\title{
Exploring the gravity sector of emergent higher-spin gravity: effective action and a solution
}

\author{
Stefan Fredenhagen ${ }^{a, b}$ and Harold C. Steinacker ${ }^{a}$ \\ ${ }^{a}$ Faculty of Physics, University of Vienna, \\ Boltzmanngasse 5, Vienna 1090, Austria \\ ${ }^{b}$ Erwin Schrödinger International Institute for Mathematics and Physics, University of Vienna, \\ Boltzmanngasse 9, Vienna 1090, Austria \\ E-mail: stefan.fredenhagen@univie.ac.at, \\ harold.steinacker@univie.ac.at
}

ABSTRACT: We elaborate the description of the semi-classical gravity sector of Yang-Mills matrix models on a covariant quantum FLRW background. The basic geometric structure is a frame, which arises from the Poisson structure on an underlying $S^{2}$ bundle over spacetime. The equations of motion for the associated Weitzenböck torsion obtained in [1] are rewritten in the form of Yang-Mills-type equations for the frame. An effective action is found which reproduces these equations of motion, which contains an Einstein-Hilbert term coupled to a dilaton, an axion and a Maxwell-type term for the dynamical frame. An explicit rotationally invariant solution is found, which describes a gravitational field coupled to the dilaton.

Keywords: M(atrix) Theories, Models of Quantum Gravity, Higher Spin Gravity

ARXIV EPRINT: 2101.07297 


\section{Contents}

1 Introduction $\quad 2$

2 Matrix model and cosmological spacetime solution 3

2.1 The background geometry 4

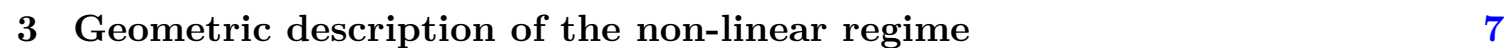

$\begin{array}{lll}3.1 & \text { Weitzenböck connection and torsion } & 7\end{array}$

3.2 Equations of motion for the torsion and frame 9

3.3 Einstein equation and geometric energy-momentum tensor 13

4 An effective action $\quad 13$

5 Relation with the Cartan formalism and (no) local Lorentz transforma$\begin{array}{ll}\text { tions } & 16\end{array}$

$\begin{array}{lll}6 & \text { Spherically symmetric static solution } & 17\end{array}$

7 Semi-classical reconstruction of the matrices $Z_{\dot{\alpha}} \quad 22$

$\begin{array}{lll}8 & \text { Discussion } & 25\end{array}$

$\begin{array}{ll}\text { A Conventions and useful formulas } & 26\end{array}$

B Divergence constraint and antisymmetric torsion $\quad 27$

$\begin{array}{ll}\text { B.1 Divergence constraint } & 27\end{array}$

$\begin{array}{ll}\text { B.2 The totally antisymmetric torsion } T^{(\mathrm{AS})} & 29\end{array}$

$\begin{array}{lll}\text { C } & \text { 6-dimensional configuration space and constraints } & 31\end{array}$

C.1 General setup 31

$\begin{array}{lll}\text { C.2 } & \mathrm{SO}(3) \text {-invariant functions and Poisson brackets } & 32\end{array}$

$\begin{array}{ll}\text { D Geometric energy-momentum tensor } & 33\end{array}$

E Geometric actions and identities $\quad 35$

E.1 Einstein-Hilbert term from torsion 35

E.2 Axion identity 36

E.3 Variation of the action $S_{T} \quad 36$ 


\section{Introduction}

Classical gravity is well described by general relativity (GR) whose dynamics is given by the Einstein-Hilbert action. However, this formulation is not well suited for quantization. Moreover, there is no straightforward way to generalize the Einstein-Hilbert action to noncommutative spaces, which are expected in a quantum theory of gravity [2]. This suggests that the Einstein-Hilbert action should only be considered as an effective action, rather than a fundamental starting point. This is indeed what happens in string theory which does lead to a similar effective gravity action, but only in critical dimensions 10 or 26. This motivates to consider matrix models such as the IIB or IKKT matrix model [3] as fundamental starting point. While this model is known to be related to critical IIB string theory, it opens up the possibility to study novel ways and mechanisms to obtain space-time and gravity on suitable noncommutative spaces or brane solutions, cf. [4-13].

In this paper, we study the non-linear dynamics of the effective gravity sector which emerges from the IKKT or IIB matrix model on a certain type of 3+1-dimensional covariant quantum space [14] (cf. [15-18]). The effective metric arises from a dynamical frame $E^{\dot{\alpha}}=$ $\left\{Z^{\dot{\alpha}},.\right\}$, which arises from the basic matrices $Z^{\dot{\alpha}}$ of the matrix model via the Poisson structure in the semi-classical limit. The semi-classical equations of the matrix model were recast in [1] as non-linear geometric equations, in terms of the torsion of the Weitzenböck connection associated to this frame. Covariance under volume-preserving diffeomorphisms is manifest in this formalism, which originates from the gauge invariance of the matrix model. The frame and all derived objects are in general higher-spin valued, somewhat reminiscent of Vasiliev-type higher-spin gauge theory $[19,20]$. The higher-spin modes arise here due to the internal structure of the brane solution as twisted $S^{2}$ bundle over space-time. The resulting 3+1-dimensional gravity theory was shown to be free of ghosts in [21], and to reproduce the Ricci-flat linearized metric perturbations of general relativity including a linearized Schwarzschild solution [22].

In the present paper, we rewrite these geometric equations for the frame in a more familiar form using the standard Levi-Civita connection. It turns out that the totally antisymmetric sector of the torsion reduces on-shell to a scalar field identified as axion, while the contraction of the torsion determines the dilaton. We obtain covariant equations of motion for all these fields, including generalized Maxwell-type equations for the frame.

Moreover, we find an effective action for these geometric quantities, in a generalized sense: the effective action reproduces these equations of motion, provided the frame, the dilaton and the axion are considered as independent quantities. This action takes a fairly familiar form involving an Einstein-Hilbert term and kinetic terms for the frame, the dilaton and the axion. This action, however, becomes a trivial identity once all constraints of the framework are used. Nevertheless, the action is expected to be useful, at least as a device to recover the geometric equations of motion in a transparent way.

The resulting gravity theory is clearly richer than GR, notably due to the presence of a dilaton and axion field, which is determined by the frame. In particular, there is no manifest local Lorentz (gauge) invariance acting on the frame. That is not a problem per se, and invariance under volume-preserving diffeos is manifest [1]. The vacuum solutions 
are not guaranteed to be Ricci-flat; however, Ricci-flatness does hold at the linearized level [22].

As a first step to understand the resulting physics at the non-linear level, we obtain in the second part of this paper an explicit solution of the non-linear geometric equations for a spherically symmetric static geometry centered at some point in space. The resulting geometry coincides with the linearized Schwarzschild geometry at the linearized level (as expected), but it deviates from it at the non-linear level, with a non-vanishing dilaton contribution. In particular there is no horizon, and the singularity at the origin is mild and integrable. Therefore this solution should presumably be interpreted as vacuum solution without matter, and its physical significance is not clear at this point; however, it illustrates how gravity is extended in the present framework. From a structural point of view, it also illustrates how the higher-spin contributions may cancel in the effective metric.

We expect that the present paper should be a useful starting point for finding further solutions of the model, and exploring the resulting theory in more depth. The paper is organized as follows: after a brief summary of the required background in section 2 , the main results are the covariant equations of motion in section 3 , the effective action in section 4 , and the new solution in sections 6 and 7 . In addition, the appendices contain a number of new identities and structural results.

\section{Matrix model and cosmological spacetime solution}

The model underlying the present paper is the IKKT or IIB matrix model [3] with mass term,

$$
S[Z, \Psi]=\operatorname{Tr}\left(\left[Z^{\dot{\alpha}}, Z^{\dot{\beta}}\right]\left[Z_{\dot{\alpha}}, Z_{\dot{\beta}}\right]+2 m^{2} Z_{\dot{\alpha}} Z^{\dot{\alpha}}+\bar{\Psi} \Gamma_{\dot{\alpha}}\left[Z^{\dot{\alpha}}, \Psi\right]\right) .
$$

We will ignore the fermionic matrices $\Psi$. Solutions of this model are then given in terms of some "matrix configurations" consisting of $9+1$ hermitian matrices $Z^{\dot{\alpha}} \in \operatorname{End}(\mathcal{H})$ for $\dot{\alpha}=0, \ldots, 9$, which satisfy the equations of motion (e.o.m.)

$$
\left[Z^{\dot{\alpha}},\left[Z_{\dot{\alpha}}, Z_{\dot{\beta}}\right]\right]=m^{2} Z_{\dot{\beta}} .
$$

Dotted indices transform under a global $\mathrm{SO}(9,1)$, and indices are raised and lowered accordingly with $\eta^{\dot{\alpha} \dot{\beta}}$. Fluctuations $Z^{\dot{\alpha}} \rightarrow Z^{\dot{\alpha}}+\mathcal{A}^{\dot{\alpha}}$ of this background are then governed by a (non-commutative) gauge theory, where the fluctuations $\mathcal{A}$ are typically viewed as functions on a background "brane" $\mathcal{M}^{(6)}$ defined by the $Z^{\dot{\alpha}}$.

We consider a class of solutions of (2.2) which are deformations of (i.e. which have similar structure as) the following solution found in [14]

$$
\begin{aligned}
Z^{\dot{\alpha}} & =\frac{1}{R} M^{\dot{\alpha} 4}, & & \dot{\alpha}=0, \ldots, 3, \\
Z^{\dot{\alpha}} & =0, & \dot{\alpha} & =4, \ldots, 9 .
\end{aligned}
$$

Here $M^{a b} \in \operatorname{End}\left(\mathcal{H}_{n}\right), a, b=0, \ldots, 5$ are certain unitary irreducible representations of $\mathfrak{s o}(4,2)$ known as doubleton series, which depend on a label $n \in \mathbb{N}$. They satisfy

$$
\left[M_{a b}, M_{c d}\right]=i\left(\eta_{a c} M_{b d}-\eta_{a d} M_{b c}-\eta_{b c} M_{a d}+\eta_{b d} M_{a c}\right)
$$


with $\eta_{a b}=\operatorname{diag}(-1,1,1,1,1,-1)$, along with some constraints. Due to these constraints, the background can be interpreted as a 6-dimensional quantized coadjoint orbit $\mathcal{M}^{(6)}$, with a natural correspondence of matrices with functions

$$
\operatorname{End}\left(\mathcal{H}_{n}\right) \cong \mathcal{C}\left(\mathcal{M}^{(6)}\right)
$$

In particular, $Z^{\dot{\alpha}}$ and the fluctuations $\mathcal{A}^{\dot{\alpha}}$ can be interpreted as (quantized) functions on $\mathcal{M}^{(6)}$. More precisely, $\mathcal{M}^{(6)}$ turns out to be a twisted sphere bundle ${ }^{1}$ over a cosmological Friedmann-Lemaître-Robertson-Walker (FLRW) spacetime,

$$
\mathcal{M}^{(6)} \stackrel{\text { loc }}{\cong} \mathcal{M}^{3,1} \times S^{2}
$$

in the sense that the local stabilizer of a point $p \in \mathcal{M}^{3,1}$ acts non-trivially on the fiber $S^{2}$. This means that non-trivial harmonics on $S^{2}$ lead to higher-spin modes on $\mathcal{M}^{3,1}$, as explained in [14] or in the introductory review [23]. The geometry will be described more explicitly below in section 2.1.

We will work in the semi-classical limit, where commutators $[.,.] \sim i\{.,$.$\} are replaced$ by Poisson brackets. Then the e.o.m. (2.2) reduces to

$$
\left\{Z_{\dot{\alpha}}, \hat{\Theta}^{\dot{\alpha} \dot{\beta}}\right\}=m^{2} Z^{\dot{\beta}}, \quad \hat{\Theta}^{\dot{\alpha} \dot{\beta}}=-\left\{Z^{\dot{\alpha}}, Z^{\dot{\beta}}\right\} .
$$

At the linearized level, the resulting higher-spin gauge theory was elaborated in [14, 21]. A suitable formalism to understand the gravity sector at the non-linear level was developed in [1], based on the Weitzenböck connection associated to the frame

$$
E_{\dot{\alpha}}=\left\{Z_{\dot{\alpha}}, .\right\}=E_{\dot{\alpha}}{ }^{\mu} \partial_{\mu}, \quad E_{\dot{\alpha}}{ }^{\mu}=\left\{Z_{\dot{\alpha}}, x^{\mu}\right\}
$$

In the present paper, we will develop this description further, and find a non-trivial solution at the non-linear level. All indices $\dot{\alpha}$ will run from 0 to 3 henceforth.

\subsection{The background geometry}

We start by reviewing the geometry of the above background or reference solution; more details can be found in [14]. The spacetime $\mathcal{M}^{3,1}$ can be described in terms of the Cartesian coordinate functions

$$
x^{\mu} \sim \tilde{R} M^{\mu 5}, \quad \mu=0, \ldots, 3
$$

where $\tilde{R}$ has dimension length. The constraints due to the doubleton representations imply

$$
\eta_{\mu \nu} x^{\mu} x^{\nu}=:-R^{2} \cosh ^{2}(\eta) \leq-R^{2}, \quad R=\frac{n}{2} \tilde{R}
$$

thereby defining the cosmic time variable $\eta$. This describes (a double cover ${ }^{2}$ of) the segment of $\mathbb{R}^{3,1}$. The equations of motion (2.2) relate $R$ to the mass parameter in the matrix model

\footnotetext{
${ }^{1}$ In more technical terms, $\mathcal{M}^{(6)}$ is an $\mathrm{SO}(3,1)$ - equivariant bundle.

${ }^{2}$ The two sheets of $\mathcal{M}^{3,1}$ describe the universe before and after the Big Bounce. We will only consider the late-time era, where the global structure is irrelevant.
} 
by $(m R)^{2}=3$. The space-like 3 -hyperboloids $H^{3}$ defined by $\eta=$ const. will be recognized as equal-time slices of a $k=-1$ FLRW space-time. The $x^{\mu}$ transform as a vector of $\mathrm{SO}(3,1)$, which should be seen as isometry group of the space-like $H^{3}$.

As indicated above, $\mathcal{M}^{3,1}$ is the base manifold of an $S^{2}$ bundle $\mathcal{M}^{(6)}$ (2.6). The fiber $S^{2}$ is described by 4 further functions

$$
t^{\mu} \sim \frac{1}{R} M^{\mu 4}, \quad \mu=0, \ldots, 3
$$

on $\mathcal{M}^{(6)}$, which also transform as vector of $\mathrm{SO}(3,1)$, subject to the constraints

$$
\begin{aligned}
t_{\mu} t^{\mu} & =\tilde{R}^{-2} \cosh ^{2}(\eta), \\
t_{\mu} x^{\mu} & =0 .
\end{aligned}
$$

Hence the $x^{\mu}, t^{\mu}$ should be viewed as functions on $\mathcal{M}^{(6)}$. At the reference point $x=$ $\left(x^{0}, 0,0,0\right)$, these relations imply $t^{0}=0$ and $t_{i} t_{i}=\tilde{R}^{-2} \cosh ^{2}(\eta)$. While $x \in \mathcal{M}^{(3,1)}$ is invariant under its local $\mathrm{SO}(3)$ stabilizer, the $t^{\mu}$ transform as vectors under this $\mathrm{SO}(3)$, leading to the structure of a twisted or equivariant bundle. Accordingly, the space of functions

$$
\mathcal{C}\left(\mathcal{M}^{(6)}\right)=\bigoplus_{s \geq 0} \mathcal{C}^{s}
$$

decomposes into higher-spin modules $\mathcal{C}^{s}$, which can be viewed as functions on $\mathcal{M}^{3,1}$ taking value in the spin $s$ representation of the local $\mathrm{SO}(3)$, given by irreducible rank $s$ polynomials in $t^{\mu}$.

Being a coadjoint orbit, the bundle space $\mathcal{M}^{(6)}$ carries a symplectic form $\omega$ which is invariant under $\mathrm{SO}(4,2)$. The corresponding Poisson structure is given by

$$
\begin{aligned}
& \left\{x^{\mu}, x^{\nu}\right\}=\theta^{\mu \nu}=-\tilde{R}^{2} R^{2}\left\{t^{\mu}, t^{\nu}\right\} \\
& \left\{t^{\mu}, x^{\nu}\right\}=\eta^{\mu \nu} \sinh (\eta) .
\end{aligned}
$$

The Poisson tensor $\theta^{\mu \nu}$ satisfies the constraints

$$
\begin{aligned}
t_{\mu} \theta^{\mu \nu} & =-\sinh (\eta) x^{\nu} \\
x_{\mu} \theta^{\mu \nu} & =-\tilde{R}^{2} R^{2} \sinh (\eta) t^{\nu} \\
\eta_{\mu \nu} \theta^{\mu \kappa} \theta^{\nu \lambda} & =R^{2} \tilde{R}^{2} \eta^{\kappa \lambda}-R^{2} \tilde{R}^{4} t^{\kappa} t^{\lambda}+\tilde{R}^{2} x^{\kappa} x^{\lambda} .
\end{aligned}
$$

Explicitly,

$$
\theta^{\mu \nu}=\frac{\tilde{R}^{2}}{\cosh ^{2}(\eta)}\left(\sinh (\eta)\left(x^{\mu} t^{\nu}-x^{\nu} t^{\mu}\right)+\epsilon^{\mu \nu \kappa \lambda} x_{\kappa} t_{\lambda}\right) .
$$

Note that the solution (2.3) amounts to $Z^{\dot{\alpha}}=t^{\dot{\alpha}}$ in the semi-classical regime. 
Frame and metric. Since fluctuations $Z^{\dot{\alpha}}+\mathcal{A}^{\dot{\alpha}}$ on a given background are governed by the action (2.1), their propagation is governed by an effective metric ${ }^{3}$ which can be extracted from the kinetic term $\operatorname{Tr}\left(\left[Z^{\dot{\alpha}}, \mathcal{A}\right]\left[Z_{\dot{\alpha}}, \mathcal{A}\right]\right)$. In the semi-classical limit, this leads to the frame (2.8). For the background solution, this frame takes the form

$$
\bar{E}_{\dot{\alpha}}=\left\{\bar{Z}_{\dot{\alpha}}, .\right\}=\bar{E}_{\dot{\alpha}}{ }^{\mu} \partial_{\mu}, \quad \bar{E}_{\dot{\alpha}}{ }^{\mu}=\left\{\bar{Z}_{\dot{\alpha}}, x^{\mu}\right\}=\sinh (\eta) \delta_{\dot{\alpha}}^{\mu}
$$

which defines the auxiliary metric

$$
\bar{\gamma}^{\mu \nu}=\eta^{\dot{\alpha} \dot{\beta}} \bar{E}_{\dot{\alpha}}^{\mu} \bar{E}_{\dot{\beta}}^{\nu}=\sinh ^{2}(\eta) \eta^{\mu \nu} .
$$

It turns out that the effective metric $\bar{G}$ which governs the kinetic term of all propagating modes is a conformal rescaling of the auxiliary metric $\bar{\gamma}^{\mu \nu}$, given by [14]

$$
\bar{G}^{\mu \nu}=\frac{1}{\bar{\rho}^{2}} \bar{\gamma}^{\mu \nu}, \quad \bar{\rho}^{2}=\rho_{M} \sqrt{|\bar{\gamma}|}^{-1}=\sinh ^{3}(\eta) .
$$

Here, $|\bar{\gamma}|$ is the absolute value of the determinant of $\left(\bar{\gamma}_{\mu \nu}\right)$, and

$$
\rho_{M}=\sinh (\eta)
$$

(in Cartesian coordinates $x^{\mu}$ ) is the density arising from the $\mathrm{SO}(4,1)$-invariant volume form $\Omega=\rho_{M} d^{4} x$ on $\mathcal{M}^{3,1}$, which originates from the symplectic volume form on $\mathcal{M}^{(6)}$. $\bar{G}^{\mu \nu}$ is a (hyperbolic, $k=-1$ ) FLRW metric, and can be written in terms of the cosmic scale factor $a(t)$ and the comoving time $t$ as

$$
d s_{\bar{G}}^{2}=\bar{G}_{\mu \nu} d x^{\mu} d x^{\nu}=-d t^{2}+a^{2}(t) d \Sigma^{2},
$$

where $d \Sigma^{2}$ is the $\mathrm{SO}(3,1)$-invariant metric on the space-like $H^{3}$. The cosmic scale parameter $a(t)$ is determined as

$$
\begin{aligned}
a(t)^{2} & =R^{2} \sinh (\eta) \cosh ^{2}(\eta) \stackrel{\eta \rightarrow \infty}{\approx} R^{2} \sinh ^{3}(\eta) \\
d t & =R \sinh (\eta)^{\frac{3}{2}} d \eta .
\end{aligned}
$$

Note that $a(t)$ also sets the curvature scale of the background. In the present paper, we will focus on the asymptotic regime $a(t) \rightarrow \infty$ i.e. $\eta \rightarrow \infty$, considering only perturbations of the geometry on scales far below the cosmic scale. Then the space-like metric $d \Sigma^{2}$ on $H^{3} \approx \mathbb{R}^{3}$ can be approximated by a flat metric near $x^{i}=0$, neglecting the cosmic curvature. We can then rewrite the Cartesian coordinates around the reference point $\xi=\left(\xi^{0}, 0,0,0\right)$ in terms of local spherical coordinates with radius

$$
\begin{aligned}
r^{2} & :=x_{i} x_{j} \delta^{i j} \ll x_{0}^{2}, \\
\eta_{\mu \nu} x^{\mu} x^{\nu} & =-x_{0}^{2}+r^{2}=-R^{2} \cosh ^{2}(\eta) .
\end{aligned}
$$

\footnotetext{
${ }^{3}$ There are various geometric structures which play different roles on noncommutative or quantum spaces. The present effective metric (which is analogous to the open string metric on branes with $B$ field) is distinct from the induced metric on noncommutative branes in target space (which is analogous to the closed string metric). The latter is related to the distribution of eigenvalues of the matrices, cf. [6, 27]. Finally, (quasi-) coherent states on quantum spaces are related to yet another metric [25, 26].
} 
In this regime, the internal sphere and the Poisson tensor are characterized by [1]

$$
\begin{aligned}
& |t| \approx \tilde{R}^{-1} \cosh (\eta) \\
& \theta^{0 i} \stackrel{\xi}{\approx} \tilde{R}^{2} R t^{i} \gg \theta^{i j} \stackrel{\xi}{\approx} \frac{\tilde{R}^{2} R}{\sinh (\eta)} \epsilon^{i j k} t^{k} \stackrel{\eta \rightarrow \infty}{\sim} \text { const. }
\end{aligned}
$$

\section{Geometric description of the non-linear regime}

We recall the geometric formalism based on (a higher-spin generalization of) the Weitzenböck connection and torsion. We will focus on local perturbations of the geometry in the asymptotic regime $\eta \rightarrow \infty$, where the dominant contributions arise from the derivations along $\mathcal{M}^{3,1}$ rather than the internal directions; for a more detailed discussion see [1].

\subsection{Weitzenböck connection and torsion}

The fundamental degrees of freedom of the matrix model are given by matrix configurations $Z_{\dot{\alpha}}$ and the associated vielbein

$$
E_{\dot{\alpha}}=\left\{Z_{\dot{\alpha}}, .\right\}, \quad E_{\dot{\alpha}}{ }^{\mu}=\left\{Z_{\dot{\alpha}}, y^{\mu}\right\}
$$

where $y^{\mu}$ are any local coordinate functions on $\mathcal{M}^{3,1}$. The inverse vielbein is defined as usual

$$
\begin{array}{ll}
E^{\dot{\alpha}}{ }_{\mu} E_{\dot{\beta}}{ }^{\mu}=\delta_{\dot{\beta}}^{\dot{\alpha}}, & \gamma^{\mu \nu}=\eta^{\dot{\alpha} \dot{\beta}} E_{\dot{\alpha}}{ }^{\mu} E_{\dot{\beta}}{ }^{\nu}, \\
E^{\dot{\alpha}}{ }_{\nu} E_{\dot{\alpha}}{ }^{\mu}=\delta_{\nu}^{\mu}, & \eta_{\dot{\alpha} \dot{\beta}}=E_{\dot{\alpha}}{ }^{\mu} E_{\dot{\beta}}{ }^{\nu} \gamma_{\mu \nu} .
\end{array}
$$

It is natural to define a Weitzenböck connection on $\mathcal{M}^{3,1}$ which respects this vielbein

$$
0=\nabla_{\nu} E_{\dot{\alpha}}^{\mu}=\partial_{\nu} E_{\dot{\alpha}}{ }^{\mu}+\Gamma_{\nu \rho}^{\mu} E_{\dot{\alpha}}{ }^{\rho}
$$

cf. [28]. This connection is automatically compatible with the metric $\nabla \gamma^{\mu \nu}=0$. For any vector field $V^{\mu}$ on $\mathcal{M}^{3,1}$ (possibly with a dependence on the fiber parameter $t^{\mu}$ corresponding to higher-spin modes), the (Weitzenböck) covariant derivative is then

$$
\nabla_{\mu} V^{\nu}=\partial_{\mu} V^{\nu}+\Gamma_{\mu \rho}^{\nu} V^{\rho}
$$

This connection is flat since the frame is parallel, $\nabla E_{\dot{\beta}}=0$. However, it typically has torsion,

$$
T[X, Y]=\nabla_{X} Y-\nabla_{Y} X-[X, Y]
$$

which can be computed as [1]

$$
\begin{aligned}
T_{\mu \nu}{ }^{\rho} & =\Gamma_{\mu \nu}{ }^{\rho}-\Gamma_{\nu \mu}{ }^{\rho} \\
T_{\mu \nu}{ }^{\dot{\alpha}} & =T_{\mu \nu}{ }^{\rho} E^{\dot{\alpha}}{ }_{\rho}=\partial_{\mu} E^{\dot{\alpha}}{ }_{\nu}-\partial_{\nu} E^{\dot{\alpha}}{ }_{\mu} .
\end{aligned}
$$


The torsion satisfies a Bianchi identity,

$$
0=\nabla_{\sigma} T_{\lambda \rho}{ }^{\mu}+\nabla_{\lambda} T_{\rho \sigma}{ }^{\mu}+\nabla_{\rho} T_{\sigma \lambda}{ }^{\mu}+T_{\lambda \rho}{ }^{\nu} T_{\nu \sigma}{ }^{\mu}+T_{\rho \sigma}{ }^{\nu} T_{\nu \lambda}{ }^{\mu}+T_{\sigma \lambda}{ }^{\nu} T_{\nu \rho}{ }^{\mu}
$$

which follows from the first Bianchi identity for a connection with zero curvature [29], or from the Jacobi identity in the matrix model [1]. Its contraction gives the identity

$$
\nabla_{\mu} T_{\lambda \rho}{ }^{\mu}=0 .
$$

In terms of the frame-valued torsion $T_{\mu \nu} \dot{\alpha}$ the Bianchi identity reads

$$
0=\partial_{\sigma} T_{\mu \nu}^{\dot{\alpha}}+\partial_{\mu} T_{\nu \sigma}^{\dot{\alpha}}+\partial_{\nu} T_{\sigma \mu}^{\dot{\alpha}} \text {. }
$$

Viewing $T_{\mu \nu} \dot{\alpha}$ as components of a two-form $T^{\dot{\alpha}}$, we see from (3.6) that it is the exterior derivative of the vielbein, and the Bianchi identity simply states that $T^{\dot{\alpha}}$ is closed,

$$
T^{\dot{\alpha}}=d E^{\dot{\alpha}}=\frac{1}{2} T_{\mu \nu}^{\dot{\alpha}} d x^{\mu} \wedge d x^{\nu}, \quad d T^{\dot{\alpha}}=0 .
$$

The Levi-Civita connection $\nabla^{(\gamma)}$ for the metric $\gamma^{\mu \nu}$ is related to the Weitzenböck connection via

$$
\begin{aligned}
\Gamma_{\mu \nu}{ }^{\rho} & =\Gamma_{\mu \nu}^{(\gamma)}{ }^{\rho}+K_{\mu \nu}{ }^{\rho} \\
\nabla_{\mu} V^{\nu} & =\nabla_{\mu}^{(\gamma)} V^{\nu}+K_{\mu \rho}{ }^{\nu} V^{\rho} .
\end{aligned}
$$

Here

$$
K_{\mu \nu}{ }^{\sigma}=\frac{1}{2}\left(T_{\mu \nu}{ }^{\sigma}+T_{\mu \nu}^{\sigma}-T_{\nu}{ }^{\sigma}{ }_{\mu}\right)=-K_{\mu}{ }^{\sigma}{ }_{\nu}
$$

is the contorsion of the Weitzenböck connection, which is antisymmetric in the last 2 indices. It carries the same information as the torsion, which can be reconstructed as $T_{\mu \nu}{ }^{\sigma}=K_{\mu \nu}{ }^{\sigma}-K_{\nu \mu}{ }^{\sigma}$. Note that all these quantities can in general take values in the higherspin algebra $\mathcal{C}$ of functions on $\mathcal{M}^{(6)}$. In particular, the torsion of the cosmic background is given by (see (7.26) in [1])

$$
\bar{T}_{\rho \sigma}^{\mu} \approx \frac{1}{a(t)^{2}}\left(\delta_{\sigma}^{\mu} \tau_{\rho}-\delta_{\rho}^{\mu} \tau_{\sigma}\right)
$$

where $\tau_{\mu}=\bar{G}_{\mu \nu} \tau^{\nu}$ and

$$
\tau=x^{\mu} \partial_{\mu}=a(t) \partial_{t}
$$

is the time-like vector field on the FLRW background.

Effective metric. Similarly to the discussion of the background geometry, the form of the kinetic term for fluctuations around a matrix model solution leads us to introduce an effective metric $G^{\mu \nu}$ (see (2.21)) that differs from $\gamma^{\mu \nu}$ by a conformal factor (see eq. (4.10) in $[1])$ :

$$
G^{\mu \nu}:=\frac{1}{\rho^{2}} \gamma^{\mu \nu}, \quad \rho^{2}=\rho_{M} \sqrt{|\gamma|}^{-1}
$$


where $\rho_{M} d^{4} y$ is the symplectic volume form. We shall therefore denote the scalar field $\rho$ as dilaton. The Levi-Civita connection $\nabla^{(G)}$ for the effective metric $G^{\mu \nu}$ is then

$$
\Gamma_{\mu \nu}^{(G)}{ }^{\sigma}=\Gamma_{\mu \nu}^{\sigma}+\delta_{\nu}^{\sigma} \rho^{-1} \partial_{\mu} \rho-\mathcal{K}_{\mu \nu}^{\sigma}
$$

Here

$$
\begin{aligned}
& \mathcal{K}_{\mu \nu}{ }^{\sigma}=K_{\mu \nu}{ }^{\sigma}+\left(G_{\mu \nu} \rho^{-1} \partial^{\sigma} \rho-\delta_{\mu}^{\sigma} \rho^{-1} \partial_{\nu} \rho\right)=-\mathcal{K}_{\mu}{ }^{\sigma}{ }_{\nu} \\
& \mathcal{T}_{\mu \nu}{ }^{\sigma}=T_{\mu \nu}{ }^{\sigma}+\rho^{-1}\left(\delta_{\nu}^{\sigma} \partial_{\mu} \rho-\delta_{\mu}^{\sigma} \partial_{\nu} \rho\right)
\end{aligned}
$$

is the Weitzenböck contorsion and torsion tensor of the effective frame

$$
\mathcal{E}^{\dot{\alpha}}{ }_{\mu}=\rho E^{\dot{\alpha}}{ }_{\mu} .
$$

This allows us to rewrite the effective Levi-Civita connection in terms of the Weitzenböck connection and the contorsion:

$$
\nabla_{\mu}^{(G)} V^{\sigma}=\nabla_{\mu} V^{\sigma}-\mathcal{K}_{\mu \nu}{ }^{\sigma} V^{\nu}+\rho^{-1} \partial_{\mu} \rho V^{\sigma}
$$

Accordingly, its indices should be raised and lowered with $G^{\mu \nu}$. To avoid any confusion, it is safer to write all connection and (con)torsion symbols with two lower and one upper index, where no ambiguity arises. Calligraphic fonts indicate the effective frame.

The Jacobi identity in the matrix model implies a relation between the trace of the torsion and the dilaton [1, lemma 5.2],

$$
T_{\mu \sigma}^{\mu}=K_{\mu \sigma}^{\mu}=\frac{2}{\rho} \partial_{\sigma} \rho
$$

For the rescaled frame resp. effective metric, we analogously have

$$
\mathcal{T}_{\mu \sigma}{ }^{\mu}=\mathcal{K}_{\mu \sigma}{ }^{\mu}=-\rho^{-1} \partial_{\sigma} \rho .
$$

\subsection{Equations of motion for the torsion and frame}

Starting from the semi-classical e.o.m. (2.7), the following e.o.m. for the torsion in vacuum was obtained in $[1]$

$$
\nabla_{\nu} T_{\rho \mu}^{\nu}+T_{\nu}^{\sigma}{ }_{\mu} T_{\sigma \rho}{ }^{\nu}=m^{2} \gamma_{\rho \mu} .
$$

The non-linear equation of motion encodes the non-linear structure of the Yang-Mills equations of motion (2.7). It can be rewritten in terms of the Levi-Civita connection using (3.20) and (3.22),

$$
\begin{aligned}
m^{2} \gamma_{\rho \mu}= & \nabla_{\nu}^{(G)} T_{\rho \mu}^{\nu}-\mathcal{K}_{\nu \rho}{ }^{\sigma} T_{\sigma \mu}^{\nu}-\mathcal{K}_{\nu \mu}{ }^{\sigma} T_{\rho \sigma}^{\nu}+T_{\nu}{ }_{\mu}{ }_{\mu} T_{\sigma \rho}{ }^{\nu} \\
= & \nabla_{\nu}^{(G)} T_{\rho \mu}^{\nu}+\frac{1}{2}\left(-T_{\nu \rho}^{\lambda} T_{\lambda \mu}^{\nu}-T_{\rho}{ }_{\nu}{ }_{\nu} T_{\lambda \mu}^{\nu}-T_{\rho \lambda}^{\nu} T_{\nu \mu}{ }^{\lambda}+T_{\rho \lambda}^{\nu} T_{\mu}{ }^{\lambda}{ }_{\nu}\right) \\
& -\rho^{-1} \partial_{\sigma} \rho\left(T_{\rho \mu}{ }^{\sigma}{ }^{\prime}+T_{\mu \rho}{ }^{\sigma}\right)+2 \rho^{-2} \partial_{\mu} \rho \partial_{\rho} \rho .
\end{aligned}
$$

Together with the Bianchi identity (3.7) (and the flatness of $\nabla$ ), this captures the dynamical content of the model. 
The antisymmetric part of the e.o.m. When we consider the e.o.m. (3.23), we observe that the right hand side is proportional to the metric and hence is a symmetric tensor. The left hand side is not automatically symmetric, and the e.o.m. can be split into two components: one that requires that the antisymmetric part of the left hand side vanishes, and the remaining symmetric part.

The antisymmetric part of (3.23) reads

$$
\nabla_{\nu}\left(T_{\rho \mu}^{\nu}-T_{\mu \rho}^{\nu}\right)+2 T_{\nu[\mu \mid}^{\sigma} T_{\sigma \mid \rho]}^{\nu}=0
$$

where the square brackets denote anti-symmetrization. When we introduce the totally antisymmetric component of the torsion as (cf. appendix B.2)

$$
T_{\rho \mu}^{(\mathrm{AS}) \nu}=T_{\rho \mu}^{\nu}+T_{\mu}{ }_{\rho}^{\nu}+T_{\rho \mu}{ }^{\nu},
$$

we can rewrite the antisymmetric e.o.m. (3.25) as

$$
\nabla_{\nu} T_{\rho \mu}^{(\mathrm{AS}) \nu}=T_{\nu[\rho}^{\sigma} T_{\mu] \sigma}^{(\mathrm{AS})}{ }^{\nu},
$$

where the contracted Bianchi identity (3.8) has been used.

The antisymmetric e.o.m. can be written in a more convenient form using the LeviCivita covariant derivative with respect to the effective metric $G$. Using the relation (3.20) between $\nabla^{(G)}$ and $\nabla$, as well as the result on the trace of the contorsion (3.22) we find

$$
\begin{aligned}
\rho^{-2} \nabla_{\nu}^{(G)}\left(\rho^{2} T_{\rho \mu}^{(\mathrm{AS}) \nu}\right) & =\nabla_{\nu} T^{(\mathrm{AS}) \nu}{ }_{\rho \mu}+2 K_{\nu[\rho \mid}{ }^{\sigma} T^{(\mathrm{AS}) \nu}{ }_{\sigma \mid \mu]} \\
& =\nabla_{\nu} T^{(\mathrm{AS}) \nu}{ }_{\rho \mu}-T_{\nu[\rho \mid}^{\sigma} T_{\mid \mu] \sigma}{ }^{(\mathrm{AS})}
\end{aligned}
$$

which vanishes due to the equation of motion. Hence

$$
\rho^{-2} \nabla_{\nu}^{(G)}\left(\rho^{2} T_{\rho \mu}^{(A S) \nu}\right)=0
$$

This means that it is consistent to set $T_{\rho \sigma \mu}^{(\mathrm{AS})}=0$, which holds for the background solution $\bar{T}$ in (3.13). We can interpret the antisymmetric part $T^{(A S) \nu}{ }_{\rho \mu}$ as the components of a 3 -form,

$$
\frac{1}{3 !} G_{\nu \nu^{\prime}} T_{\rho \mu}^{(A S) \nu^{\prime}} d x^{\nu} \wedge d x^{\rho} \wedge d x^{\mu}=\rho^{2} T^{\dot{\alpha}} \wedge E_{\dot{\alpha}} .
$$

The equation of motion (3.30) of $T^{(\mathrm{AS})}$ can then be rewritten — via the Hodge star with respect to $G$ - as

$$
* d *\left(\rho^{4} T^{\dot{\alpha}} \wedge E_{\dot{\alpha}}\right)=0 .
$$

Expressing $T^{(A S) \nu}{ }_{\rho \mu}$ in terms of the $*$-dual 1-form $T=T_{\sigma} d x^{\sigma}$,

$$
T_{\rho \mu}^{(\mathrm{AS}){ }_{\rho \mu}}=:-\sqrt{|G|} G^{\nu \nu^{\prime}} \varepsilon_{\nu^{\prime} \rho \mu \sigma} G^{\sigma \sigma^{\prime}} T_{\sigma^{\prime}} \quad \Longleftrightarrow \quad \rho^{2} T^{\dot{\alpha}} \wedge E_{\dot{\alpha}}=* T,
$$

the equation of motion (3.30) for $T^{(\mathrm{AS})}$ becomes

$$
\begin{aligned}
0 & =\rho^{-2} \nabla_{\nu}^{(G)}\left(\sqrt{|G|} G^{\nu \nu^{\prime}} \varepsilon_{\nu^{\prime} \rho \mu \sigma} \rho^{2} G^{\sigma \sigma^{\prime}} T_{\sigma}\right) \\
& =\rho^{-2} \sqrt{|G|} G^{\sigma \sigma^{\prime}} G^{\nu \nu^{\prime}} \varepsilon_{\nu \rho \mu \sigma} \partial_{\nu^{\prime}}\left(\rho^{2} T_{\sigma}\right) .
\end{aligned}
$$


In terms of differential forms this relation simply reads

$$
d\left(\rho^{2} T\right)=0 .
$$

This in turn means that $T_{\mu}$ can be written on-shell as

$$
\rho^{2} T_{\mu}=\partial_{\mu} \tilde{\rho}
$$

in terms of a scalar field $\tilde{\rho}$, which will be denoted as axion, for reasons that will become clear below. Hence the anti-symmetric part of the e.o.m. for the torsion reduces the 4 dof of $T^{(\mathrm{AS})}$ to the scalar field $\tilde{\rho}$, while the remaining 3 dof of the general frame disappear on-shell.

The Bianchi identity (3.10) for $T^{\dot{\alpha}}$ implies

$$
* d *\left(\rho^{-2} T\right)=* d\left(T^{\dot{\alpha}} \wedge E_{\dot{\alpha}}\right)=*\left(T^{\dot{\alpha}} \wedge T_{\dot{\alpha}}\right) .
$$

Expressing $T_{\mu}$ on-shell in terms of $\tilde{\rho}$ via (3.36) results in

$$
* d *\left(\rho^{-4} d \tilde{\rho}\right)=*\left(T^{\dot{\alpha}} \wedge T_{\dot{\alpha}}\right),
$$

or, in components (for an explicit calculation see (B.22)),

$$
\nabla_{(G)}^{\mu}\left(\rho^{-4} \partial_{\mu} \tilde{\rho}\right)=\frac{1}{4} \sqrt{|G|}^{-1} \varepsilon^{\nu \rho \mu \kappa} T_{\nu \rho}{ }^{\dot{\alpha}} T_{\kappa \mu \dot{\alpha}} .
$$

Thus $\tilde{\rho}$ is recognized as an axion-like field.

Eom for $\boldsymbol{\rho}$. Taking the trace of the equation of motion (3.23) for $T$ yields

$$
\gamma^{\nu \sigma} \nabla_{\sigma} T_{\nu \rho}{ }^{\rho}+\gamma^{\mu \rho} T_{\nu}{ }^{\sigma}{ }_{\mu} T_{\sigma \rho}{ }^{\nu}=4 m^{2} .
$$

We now rewrite $\gamma$ in terms of the effective metric $G$ via (3.15), and express $\nabla$ in terms of $\nabla^{(G)}$ using (3.20). Using the results (3.21) and (3.22) for the trace of the (con-)torsion, we obtain

$$
-2 \rho^{2} G^{\mu \nu} \nabla_{\mu}^{(G)}\left(\rho^{-1} \partial_{\nu} \rho\right)+\rho^{2} G^{\mu \rho} T_{\nu}{ }^{\sigma}{ }_{\mu} T_{\sigma \rho}{ }^{\nu}=4 m^{2} .
$$

Finally, rewriting the quadratic term in the torsion using (B.19) and replacing $T_{\mu}$ by its on-shell value (3.36), we arrive at (cf. (5.50) in [1])

$$
-\nabla_{(G)}^{\mu}\left(\rho^{-1} \partial_{\mu} \rho\right)=2 \rho^{-2} m^{2}+\frac{1}{4} T_{\mu}{ }^{\sigma}{ }_{\rho} T_{\nu \sigma}{ }^{\rho} G^{\mu \nu}+\frac{1}{2} \rho^{-4} G^{\mu \nu} \partial_{\mu} \tilde{\rho} \partial_{\nu} \tilde{\rho} .
$$

We have thus identified two scalar fields $\rho$ and $\tilde{\rho}$ which encode the trace and the totally anti-symmetric components of the torsion tensor, respectively. Both satisfy second-order equations of motion sourced by the torsion. This reflects the fact that in contrast to general relativity, there is no local Lorentz invariance for the frame. In the present theory, the frame is a physical object which satisfies the divergence constraint

$$
\nabla_{\nu}^{(G)}\left(\rho^{-2} E_{\dot{\alpha}}{ }^{\nu}\right)=0
$$

which is a consequence of the relation (3.21) between the contraction of the torsion and the dilaton (as shown in appendix B.1), and gives rise to extra physical degrees of freedom encoded in $\rho$ and $\tilde{\rho}$. These have no counterpart in general relativity. Similar fields are known to arise in various generalizations of general relativity [30-32]. 
Equation for frame-valued torsion. The equation of motion (3.23) for the torsion can be rewritten in terms of the frame-valued torsion $T_{\mu \nu}{ }^{\dot{\alpha}}=T_{\mu \nu}{ }^{\sigma} E^{\dot{\alpha}}{ }_{\sigma}$,

$$
\gamma^{\nu \nu^{\prime}} \nabla_{\nu^{\prime}} T_{\nu \rho}^{\dot{\alpha}}+T_{\sigma \rho}{ }^{\nu} T_{\nu}{ }^{\sigma \dot{\alpha}}=m^{2} E_{\rho}^{\dot{\alpha}},
$$

where one has used that the vielbein is covariantly constant.

This equation can be expressed in terms of the Levi-Civita connection with respect to the effective metric as follows

$$
\gamma^{\nu \nu^{\prime}}\left(\nabla_{\nu^{\prime}}^{(G)} T_{\nu \rho}{ }^{\dot{\alpha}}-\mathcal{K}_{\nu^{\prime} \rho}{ }^{\sigma} T_{\nu \sigma}{ }^{\dot{\alpha}}+\rho^{-1} \partial_{\nu^{\prime}} \rho T_{\nu \rho}{ }^{\dot{\alpha}}\right)+T_{\sigma \rho}{ }^{\nu} T_{\nu}{ }^{\sigma \dot{\alpha}}=m^{2} E_{\rho}^{\dot{\alpha}}
$$

using (3.22). The second term can be rewritten using

$$
-\gamma^{\nu \nu^{\prime}} \mathcal{K}_{\nu^{\prime} \rho}^{\sigma} T_{\nu \sigma}{ }^{\dot{\alpha}}+T_{\rho}^{\sigma}{ }^{\nu} T_{\nu \sigma}{ }^{\dot{\alpha}}=-\frac{1}{2} \rho^{2} G^{\nu \nu^{\prime}} T_{\nu^{\prime} \rho}^{(\mathrm{AS})}{ }^{\sigma} T_{\nu \sigma}{ }^{\dot{\alpha}}-\rho G^{\sigma \sigma^{\prime}} \partial_{\sigma^{\prime}} \rho T_{\rho \sigma}{ }^{\dot{\alpha}}
$$

where $G^{\nu \nu^{\prime}}=\rho^{-2} \gamma^{\nu \nu^{\prime}}$, which results in

$$
\rho^{-2} \nabla_{(G)}^{\nu}\left(\rho^{2} T_{\nu \rho}^{\dot{\alpha}}\right)=\frac{1}{2} G^{\nu \nu^{\prime}} T_{\nu^{\prime} \rho}^{(\mathrm{AS})} T_{\nu \sigma}{ }^{\dot{\alpha}}+\rho^{-2} m^{2} E_{\rho}^{\dot{\alpha}} .
$$

Expressing $T^{(\mathrm{AS})}$ in terms of the dual 1-form $T$ (see (B.15)), we obtain (using (A.2))

$$
\nabla_{(G)}^{\nu}\left(\rho^{2} T_{\nu \rho}^{\dot{\alpha}}\right)=\frac{1}{2} \rho^{2} \sqrt{|G|}^{-1} \varepsilon^{\nu \sigma \rho^{\prime} \mu} G_{\rho \rho^{\prime}} T_{\mu} T_{\nu \sigma^{\prime}}^{\dot{\alpha}}+m^{2} E_{\rho}^{\dot{\alpha}},
$$

or, using the on-shell relation (3.36) for $T_{\mu}$,

$$
\nabla_{(G)}^{\nu}\left(\rho^{2} T_{\nu \rho}^{\dot{\alpha}}\right)=\frac{1}{2} \sqrt{|G|}^{-1} \varepsilon^{\nu \sigma \rho^{\prime} \mu} G_{\rho \rho^{\prime}} \partial_{\mu} \tilde{\rho} T_{\nu \sigma}^{\dot{\alpha}}+m^{2} E_{\rho}^{\dot{\alpha}} .
$$

In terms of differential forms (see (3.10)), this equation can be written concisely as

$$
d\left(\rho^{2} \star T^{\dot{\alpha}}\right)=d \tilde{\rho} \wedge T^{\dot{\alpha}}+m^{2} \star E^{\dot{\alpha}} .
$$

This can easily be verified for the cosmic background solution. If the derivatives $\partial \rho \approx 0 \approx$ $\partial \tilde{\rho}$ vanish or are negligible, this reduces to the linear equation for the torsion

$$
\nabla_{(G)}^{\nu} T_{\nu \mu}^{\dot{\alpha}}=\frac{m^{2}}{\rho^{2}} E_{\mu}^{\dot{\alpha}}
$$

which has the structure of Maxwell equations for each frame index $\dot{\alpha}$. Note that $\frac{m^{2}}{\bar{\rho}^{2}}=\frac{3}{a^{2}(t)}$ is the cosmic curvature scale, so that the r.h.s. is often negligible. This equation can be rewritten as harmonic equation for the frame $E^{\dot{\alpha}}{ }_{\nu}$

$$
\Delta_{(G)} E^{\dot{\alpha}}{ }_{\nu}-\rho^{-2} \mathcal{R}_{\nu \sigma} E^{\dot{\alpha} \sigma}=\frac{m^{2}}{\rho^{2}} E^{\dot{\alpha}}{ }_{\nu}
$$

where $\Delta_{(G)}=\nabla_{(G)}^{\mu} \nabla_{\mu}^{(G)}$, dropping again $\partial \rho \approx 0 \approx \partial \tilde{\rho}$ and using the divergence constraint (3.43). These are reminiscent of Maxwell equations for the vector potential. 


\subsection{Einstein equation and geometric energy-momentum tensor}

The Ricci tensor for the effective metric $G_{\mu \nu}$ can be expressed in terms of the torsion and the dilaton. Using the e.o.m. (3.23) for the torsion, the following relation was derived in [1]

$$
\mathcal{R}_{\mu \nu}-\frac{1}{2} G_{\mu \nu} \mathcal{R}=\mathbf{T}_{\mu \nu}
$$

(absorbing a factor $8 \pi$ for convenience) where $\mathbf{T}_{\mu \nu}$ is the effective energy-momentum tensor associated to the torsion,

$$
\begin{aligned}
\mathbf{T}_{\mu \nu}= & -\frac{1}{2}\left(T_{\rho}{ }_{\nu}{ }_{\nu} T_{\mu \delta}{ }^{\rho}+T_{\rho}{ }^{\delta}{ }_{\mu} T_{\nu \delta}{ }^{\rho}\right)-K_{\delta}{ }^{\rho}{ }_{\mu} K_{\rho}{ }^{\delta}{ }_{\nu}+2 \rho^{-2} \partial_{\mu} \rho \partial_{\nu} \rho \\
& +G_{\mu \nu}\left(-\frac{1}{4} T^{\sigma \delta \rho} T_{\delta \rho \sigma}+\frac{1}{8} T^{\delta \sigma \rho} T_{\delta \sigma \rho}-\rho^{-2} \partial \rho \cdot \partial \rho-3 R^{-2} \rho^{-2}\right) .
\end{aligned}
$$

As shown in appendix D (see (D.6)), this can be written more succinctly as

$$
\mathbf{T}_{\mu \nu}=\mathbf{T}_{\mu \nu}\left[E^{\dot{\alpha}}\right]+\mathbf{T}_{\mu \nu}[\rho]+\mathbf{T}_{\mu \nu}[\tilde{\rho}]-\rho^{-2} m^{2} G_{\mu \nu}
$$

in terms of contributions of the dilaton $\rho$, the axion $\tilde{\rho}$, and a Maxwell-like contribution from the frame fields $E^{\dot{\alpha}}$. This shows again that the frame is physical, and acts - together with the dilaton and the axion - as source of the Einstein equations. The contributions $\mathbf{T}_{\mu \nu}\left[E^{\dot{\alpha}}\right]$ and $\mathbf{T}_{\mu \nu}[\tilde{\rho}]$ of the frame and the axion turn out to have slightly non-standard form, and Ricci-flat geometries may arise if the various contributions cancel. While this may seem unlikely at first sight, it was shown previously that the standard Ricci-flat solutions arise indeed in the linearized regime [14, 22], and they are expected to arise more generally in the absence of axions and dilatons in view of (3.52).

\section{An effective action}

Even though $E^{\dot{\alpha}}, \rho$ and the metric $G_{\mu \nu}$ are mutually related, let us consider them as independent quantities for the moment. Then all solutions of the above equations of motion are critical points of the following effective action

$$
S_{\mathrm{eff}}[E, G, \rho]=c_{\mathcal{R}} S_{\mathcal{R}}+c_{E} S_{E}+c_{T} S_{T}+c_{\rho} S_{\rho}+c_{m} S_{m}+c_{\tilde{m}} S_{\tilde{m}}
$$

where

$$
\begin{aligned}
S_{\mathcal{R}} & =\int d^{4} x \sqrt{|G|} \mathcal{R}[G] \\
S_{E} & =\int d^{4} x \sqrt{|G|} \rho^{2} G^{\nu \nu^{\prime}} G^{\sigma \sigma^{\prime}} T_{\nu \sigma}{ }^{\dot{\alpha}} T_{\nu^{\prime} \sigma^{\prime} \dot{\alpha}}=2 \int \rho^{2} d E^{\dot{\alpha}} \wedge \star d E_{\dot{\alpha}} \\
S_{T} & =\int d^{4} x \sqrt{|G|} G^{\mu \nu} T_{\mu} T_{\nu} \\
S_{\rho} & =\int d^{4} x \sqrt{|G|} G^{\mu \nu} \rho^{-2} \partial_{\mu} \rho \partial_{\nu} \rho \\
S_{m} & =\int d^{4} x \sqrt{|G|} m^{2} E^{\dot{\alpha}}{ }_{\kappa} E_{\dot{\alpha} \kappa^{\prime}} G^{\kappa \kappa^{\prime}} \\
S_{\tilde{m}} & =\int d^{4} x \sqrt{|G|} m^{2} \rho^{-2} .
\end{aligned}
$$


Here $G, E$ and $\rho$ are considered as independent objects which define the torsion $T_{\mu \nu}{ }^{\dot{\alpha}}$, and $T_{\mu}$ is defined as $T=\star T^{(\mathrm{AS})}$ (B.14). The variations of these terms are as follows

$$
\begin{aligned}
\delta S_{\mathcal{R}}= & \int d^{4} x \sqrt{|G|}\left(\mathcal{R}_{\mu \nu}-\frac{1}{2} G_{\mu \nu} \mathcal{R}\right) \delta G^{\mu \nu} \\
\delta S_{E}= & \int d^{4} x \sqrt{|G|}\left(-2 \delta G^{\mu \nu} \mathbf{T}_{\mu \nu}\left[E^{\dot{\alpha}}\right]-4 \nabla^{\nu}\left(\rho^{2} G^{\kappa \kappa^{\prime}} T_{\nu \kappa}{ }^{\dot{\alpha}}\right) \delta E_{\dot{\alpha} \kappa^{\prime}}\right. \\
& \left.+2 G^{\nu \nu^{\prime}} T_{\nu \sigma}{ }^{\dot{\alpha}} T_{\nu^{\prime}}{ }_{\dot{\alpha}} \rho^{-1} \delta \rho\right) \\
\delta S_{T}= & \int d^{4} x \sqrt{|G|}\left(-2 \delta G^{\mu \nu} \mathbf{T}^{(\mathrm{AS})}{ }_{\mu \nu}[T]+4 T \cdot T \rho^{-1} \delta \rho\right) \\
& -2 \int d^{4} x \rho^{2} \delta E_{\dot{\alpha} \mu}\left(T_{\kappa} \varepsilon^{\nu \mu \sigma \kappa} T_{\nu \sigma}{ }^{\dot{\alpha}}+E^{\dot{\alpha}}{ }_{\sigma} \varepsilon^{\nu \mu \sigma \kappa} \rho^{-2} \partial_{\nu}\left(\rho^{2} T_{\kappa}\right)\right) \\
\delta S_{\rho}= & \int d^{4} x \sqrt{|G|}\left(\frac{1}{2} \delta G^{\mu \nu} \mathbf{T}_{\mu \nu}[\rho]-2 \nabla_{(G)}^{\mu}\left(\rho^{-1} \partial_{\mu} \rho\right) \rho^{-1} \delta \rho\right) \\
\delta S_{m}= & \int d^{4} x \sqrt{|G|}\left(-m^{2} \rho^{-2} G_{\mu \nu} \delta G^{\mu \nu}+2 m^{2} G^{\mu \nu} E^{\dot{\alpha}}{ }_{\mu} \delta E_{\dot{\alpha} \nu}\right) \\
\delta S_{\tilde{m}}= & \int d^{4} x \sqrt{|G|}\left(-\frac{1}{2} m^{2} \rho^{-2} G_{\mu \nu} \delta G^{\mu \nu}-2 m^{2} \rho^{-3} \delta \rho\right)
\end{aligned}
$$

using the results of appendix D. The coefficient of $\delta \rho$ coincides with the e.o.m. (3.42) if

$$
c_{\rho}=-4 c_{E}, \quad c_{T}=c_{E}, \quad c_{\tilde{m}}=-8 c_{E} .
$$

The coefficient of $\delta G^{\mu \nu}$ coincides with the e.o.m. (3.53) and using (3.55) if

$$
c_{E}=\frac{1}{2} c_{\mathcal{R}}, \quad c_{T}=\frac{1}{2} c_{\mathcal{R}}, \quad c_{\rho}=-2 c_{\mathcal{R}}, \quad c_{m}=c_{\mathcal{R}} .
$$

Finally, the coefficient of $\delta E^{\dot{\alpha}}$ vanishes as a consequence of the e.o.m. (3.48) if

$$
c_{T}=c_{E} .
$$

Remarkably, all these conditions are compatible. Therefore all critical points of the semiclassical matrix model are critical points of

$$
S_{\text {eff }}[E, G, \rho]=2 S_{\mathcal{R}}+S_{E}+S_{T}-4 S_{\rho}+2 S_{m}-8 S_{\tilde{m}}
$$

for independent variations of $E, G$ and $\rho$. This result should be useful to understand better the present gravity theory. For example, it provides an "explanation" for (or at least a book-keeping of) the effective Einstein equation (3.53) and the explicit form of the contributions (3.55) on the r.h.s.

It should be noted that the sign of $S_{E}$ is non-standard, which is reflected in the negative sign in the energy-momentum tensor associated to the frame (D.10). This looks "wrong" at first sight, but remember that the $E^{\dot{\alpha}}$ does not play the role of a vector field coupled to gravity, it rather defines the metric. This is very different from the usual role of a vector field in gravity, and it was shown in [21] that no ghosts arise in this theory, at least at the linearized level. 
Extra gauge invariance. Note that the unrestricted action $S_{\text {eff }}[E, G, \rho]$ enjoys the gauge invariance

$$
E_{\mu}^{\dot{\alpha}} \rightarrow E_{\mu}^{\dot{\alpha}}+\partial_{\mu} \Lambda^{\dot{\alpha}}
$$

subject to the constraint

$$
0=E^{\dot{\alpha} \mu} \partial_{\mu} \Lambda_{\dot{\alpha}}
$$

(sum over $\dot{\alpha}$ !), which guarantees that the mass term $S_{m}$ is invariant. We shall not pursue this observation any further here.

Alternative action using the axion. Now we impose the e.o.m. (3.34) for the totally antisymmetric part of the torsion, replacing $T_{\mu} \rightarrow \rho^{-2} \partial_{\mu} \tilde{\rho}$ according to (3.36). If we consider $\tilde{\rho}$ as independent quantity, its e.o.m. (3.39) is recovered if we replace the term $S_{T}$ by

$$
S_{T} \rightarrow c_{\tilde{\rho}} S_{\tilde{\rho}}+c_{\tilde{E}} S_{\tilde{E}}
$$

where

$$
\begin{aligned}
S_{\tilde{\rho}} & =\int d^{4} x \sqrt{|G|} G^{\mu \nu} \rho^{-4} \partial_{\mu} \tilde{\rho} \partial_{\nu} \tilde{\rho} \\
S_{\tilde{E}} & =\int d^{4} x \tilde{\rho} \varepsilon^{\nu \sigma \mu \kappa} T_{\nu \sigma}{ }^{\dot{\alpha}} T_{\mu \kappa \dot{\alpha}}=4 \int \tilde{\rho} d E^{\dot{\alpha}} \wedge d E_{\dot{\alpha}} .
\end{aligned}
$$

Hence $\tilde{\rho}$ is recognized as axion associated to the frame field. The variations are

$$
\begin{aligned}
\delta S_{\tilde{\rho}} & =\int d^{4} x \sqrt{|G|}\left(-2 \nabla_{(G)}^{\mu}\left(\rho^{-4} \partial_{\mu} \tilde{\rho}\right) \delta \tilde{\rho}-4 G^{\mu \nu} \rho^{-5} \partial_{\mu} \tilde{\rho} \partial_{\nu} \tilde{\rho} \delta \rho+2 \mathbf{T}^{\mu \nu}[\tilde{\rho}] \delta G^{\mu \nu}\right) \\
\delta S_{\tilde{E}} & =\int d^{4} x\left(4 \varepsilon^{\nu \sigma \mu \kappa} T_{\nu \sigma}^{\dot{\alpha}} \partial_{\kappa} \tilde{\rho} \delta E_{\mu \dot{\alpha}}-\varepsilon^{\nu \sigma \kappa \mu} T_{\nu \sigma}^{\dot{\alpha}} T_{\mu \kappa \dot{\alpha}} \delta \tilde{\rho}\right) .
\end{aligned}
$$

The $\delta \tilde{\rho}$ terms reproduce the e.o.m. (3.39) for $\tilde{\rho}$ if $c_{\tilde{E}}=-\frac{1}{2} c_{\tilde{\rho}}$. The coefficient of $\delta \rho$ agrees with that of $S_{T}$ if $c_{\tilde{\rho}}=-c_{T}$, and the remaining equations of motion are also satisfied if $c_{T}=2 c_{\tilde{E}}$. Therefore all critical points of the semi-classical matrix model are critical points of

$$
S_{\mathrm{eff}}[E, G, \rho, \tilde{\rho}]=2 S_{\mathcal{R}}+S_{E}-S_{\tilde{\rho}}+\frac{1}{2} S_{\tilde{E}}-4 S_{\rho}+2 S_{m}-8 S_{\tilde{m}}
$$

for independent variations of $E, G, \rho$ and $\tilde{\rho}$. This is somewhat reminiscent of axion-dilaton gravity, cf. [30-32], though the kinetic term for the frame is distinct.

Reduced action and triviality. Now we impose the constraint (3.15)

$$
G^{\mu \nu}=\rho^{-2} E^{\dot{\alpha} \mu} E_{\dot{\alpha}}{ }^{\nu}
$$

Imposing also the divergence constraint, we can use the identity (E.4)

$$
S_{E}=4\left(S_{\mathcal{R}}-6 S_{\rho}\right)
$$


Then

$$
\begin{aligned}
S_{\text {eff }}[E] & =c_{\mathcal{R}} S_{\mathcal{R}}+c_{E} 4\left(S_{\mathcal{R}}-6 S_{\rho}\right)+c_{T} S_{T}+c_{\rho} S_{\rho}+c_{m} S_{m} \\
& =c_{\mathcal{R}}\left(3 S_{\mathcal{R}}+\frac{1}{2} S_{T}-14 S_{\rho}\right)+c_{m} S_{m} .
\end{aligned}
$$

This action is trivial: the first term vanishes due to the identity (E.6)

$$
3 S_{\mathcal{R}}=14 S_{\rho}-\frac{1}{2} S_{T},
$$

and the mass term is topological in view of (A.8). This means that the effective action becomes trivial if all constraints are imposed. Even though this may be disappointing, it is in line with the notorious difficulty of obtaining a geometric action for higher-spin gravity. The present matrix-model framework does provide such an action, where the frame is obtained as derivative of the (semi-classical) matrix degrees of freedom. The geometric actions (4.7) and (4.13) only serve as effective actions for an extended configuration space where the geometrical quantities are viewed as independent.

\section{Relation with the Cartan formalism and (no) local Lorentz transfor- mations}

It is well-known that for any frame $\mathcal{E}_{\dot{\alpha} \nu}$ associated to a given metric $G_{\mu \nu}$, one can act with a local Lorentz transformation on the frame as follows

$$
\mathcal{E}_{\dot{\alpha} \nu}(x) \rightarrow \Lambda_{\dot{\alpha}}^{\dot{\beta}}(x) \mathcal{E}_{\dot{\beta} \nu}(x)
$$

leading to the same metric. In this sense the frames form an $\mathrm{SO}(3,1)$ bundle over spacetime, leading to the Cartan formalism with a spin connection as discussed further below. However, in the present setting, the frame $E_{\dot{\beta}}=\rho^{-1} \mathcal{E}_{\dot{\beta}}$ satisfies the divergence constraint (3.43), as well as the on-shell relation (3.34). This means that the above local Lorentz (gauge) symmetry is broken, and the extra degrees of freedom of the frame encode the dilaton $\rho$ and the axion $\tilde{\rho}$. This is not a problem, but it exhibits a fundamental difference between general relativity and the present theory, where the frame is a fundamental object. The dilaton $\rho$ is also related to the invariant symplectic volume via (A.8), leading to a reduction of the diffeomorphism invariance to the volume-preserving diffeos (B.12).

We should therefore ask the following question: given some metric $G_{\mu \nu}$, can we always find a gauge (i.e. a representative in the frame bundle) and functions $\rho$ and $\tilde{\rho}$ such that $E_{\dot{\beta}}=\rho^{-1} \mathcal{E}_{\dot{\beta}}$ satisfies the divergence constraint (3.43) as well as the conditions (3.36)? By counting degrees of freedom (d.o.f.), it is plausible that the answer should be generically yes. Indeed, if we consider $\rho$ and $\tilde{\rho}$ as independent fields, then the 6 d.o.f. of $\Lambda_{\dot{\alpha}}^{\dot{\beta}}(x)$ together with the 2 d.o.f. $\rho$ and $\tilde{\rho}$ should allow to satisfy the $4+4$ equations (3.36) and (3.43). But this means that both the dilaton $\rho$ and the axion $\tilde{\rho}$ are determined by the given metric $G_{\mu \nu}$. This is reflected in relations such as (D.5). In particular they do not add any propagating degrees of freedom. This is consistent with the results in $[14,21]$ for the linearized case, where it was shown that the only physical, propagating modes are those encoded in the degrees of freedom of a (massive) graviton. A more detailed understanding of $\rho, \tilde{\rho}$ and their relation to the geometry should be developed elsewhere. 
Relation with the Cartan structure equations. To make contact with the standard Cartan formalism of general relativity, we consider the (co-)frame (3.19) $\mathcal{E}_{\dot{\beta}}=\rho E_{\dot{\beta}}=$ $\mathcal{E}_{\dot{\beta} \mu} d x^{\mu}$. Then the spin connection one-form $\omega_{\dot{\alpha} \dot{\beta}}=\omega_{\mu \dot{\alpha} \dot{\beta}} d x^{\mu}=-\omega_{\dot{\beta} \dot{\alpha}}$ satisfies the first Cartan structure equations

$$
d \mathcal{E}_{\dot{\alpha}}=-\omega_{\dot{\alpha}}^{\dot{\beta}} \wedge \mathcal{E}_{\dot{\beta}}
$$

Clearly this is closely related to the (con)torsion of the Weitzenböck connection. Indeed,

$$
d \mathcal{E}_{\dot{\alpha}}=\frac{1}{2}\left(\partial_{\mu} \mathcal{E}_{\dot{\alpha} \nu}-\partial_{\nu} \mathcal{E}_{\dot{\alpha} \mu}\right) d x^{\mu} \wedge d x^{\nu}=-\omega_{\mu \dot{\alpha}}^{\dot{\beta}} \mathcal{E}_{\dot{\beta} \nu} d x^{\mu} \wedge d x^{\nu}=\frac{1}{2} \mathcal{T}_{\mu \nu \dot{\alpha}} d x^{\mu} \wedge d x^{\nu}
$$

so that

$$
\mathcal{T}_{\mu \nu \dot{\alpha}}=-\omega_{\mu \dot{\alpha}}^{\dot{\beta}} \mathcal{E}_{\dot{\beta} \nu}+\omega_{\nu \dot{\alpha}}^{\dot{\beta}} \mathcal{E}_{\dot{\beta} \mu}=\omega_{\nu \dot{\alpha} \mu}-\omega_{\mu \dot{\alpha} \nu}
$$

This provides the relation of the spin connection $\omega$ of the frame bundle to the torsion tensor $\mathcal{T}$ of the Weitzenböck connection. Even though the spin connection is not a tensor in the Cartan formalism due to the local Lorentz transformations, $\mathcal{T}_{\mu \nu \dot{\alpha}}$ is a tensor in the present formalism, where the frame is physical. Hence $\mathcal{T}_{\mu \nu \dot{\alpha}}$ can be viewed as a physical manifestation of the spin connection in the Cartan formulation of Riemannian geometry.

\section{Spherically symmetric static solution}

In this section, we will find a simple rotationally invariant static solution of the nonlinear equations of motion for the frame.

SO(3)-invariant frames. We are interested in local perturbations of the background which are centered at $x^{i}=0$, hence at $r=0$, and invariant under (global) $\mathrm{SO}(3)$ rotations. We first observe that the background frame $\bar{E}_{\dot{\alpha}}{ }^{\mu}=\sinh (\eta) \delta_{\dot{\alpha}}^{\mu}(2.19)$ is $\mathrm{SO}(3)$-invariant if the frame index $\dot{\alpha}$ is transformed as a vector. This is the manifest global $\mathrm{SO}(3)$ symmetry of the matrix model, and it seems natural to keep this $\mathrm{SO}(3)$ symmetry manifest for the perturbed rotationally-invariant geometry. This is easily achieved in Cartesian coordinates $x^{\mu}$, adopting the notation ${ }^{4}$

$$
t=x^{0}, \quad r^{2}=x^{i} x^{j} \delta_{i j}
$$

Then the most general spherically symmetric ansatz for the frame $E^{\dot{\alpha}}{ }_{\mu}$ is

$$
\begin{aligned}
E_{0}^{0} & =A, \\
E_{0}^{i} & =E x^{i}, \\
E^{0}{ }_{i} & =D x^{i}, \\
E^{i}{ }_{j} & =F x^{i} x^{j}+\delta^{i}{ }_{j} B+S \epsilon_{i j m} x^{m},
\end{aligned}
$$

\footnotetext{
${ }^{4}$ The $t$ defined here should not be confused with the comoving time that was introduced in section 2.1 and which will not be used in the current section.
} 
where $A, B, D, E, F$ and $S$ are assumed to be functions of $r$ only. In particular we consider only static ${ }^{5}$ configurations. We can eliminate $D$ and $F$ using a simple change of coordinates $t \rightarrow f(r)+t$ and $x^{i} \rightarrow g(r) x^{i}$, which is understood from now on. In terms of differential forms $E^{\dot{\alpha}}=E^{\dot{\alpha}}{ }_{\mu} d x^{\mu}$, the (co)frame is then

$$
E^{0}=A d t, \quad E^{i}=B d x^{i}+E x^{i} d t+S \epsilon_{i j m} x^{m} d x^{j}
$$

and the associated torsion 2-form $T^{\dot{\alpha}}=d E^{\dot{\alpha}}$ is obtained as

$$
\begin{aligned}
& T^{0}=d E^{0}=A^{\prime} d r \wedge d t, \\
& T^{i}=d E^{i}=B^{\prime} d r \wedge d x^{i}+E d x^{i} \wedge d t+x^{i} E^{\prime} d r \wedge d t+S \epsilon_{i j m} d x^{m} \wedge d x^{j}+S^{\prime} \epsilon_{i j m} x^{m} d r \wedge d x^{j} .
\end{aligned}
$$

It is easy to see that the totally antisymmetric part $T^{(\mathrm{AS})}$ of the torsion vanishes if $S=0$, which we assume henceforth. Then

$$
T_{\mu}=0, \quad \tilde{\rho}=\text { const. },
$$

and the frame is given in matrix form by

$$
E_{\mu}^{\dot{\alpha}}=\left(\begin{array}{cccc}
A & 0 & 0 & 0 \\
E x^{1} & B & 0 & 0 \\
E x^{2} & 0 & B & 0 \\
E x^{3} & 0 & 0 & B
\end{array}\right) .
$$

The inverse frame is

$$
E_{\dot{\alpha}}{ }^{\mu}=\left(\begin{array}{cccc}
A^{-1} & -\frac{E}{A B} x^{1} & -\frac{E}{A B} x^{2} & -\frac{E}{A B} x^{3} \\
0 & B^{-1} & 0 & 0 \\
0 & 0 & B^{-1} & 0 \\
0 & 0 & 0 & B^{-1}
\end{array}\right)
$$

and the metric obtained from this frame reads

$$
\begin{aligned}
\gamma_{00} & =-A^{2}+r^{2} E^{2}, \\
\gamma_{0 i} & =B E x^{i}, \\
\gamma_{i j} & =\delta_{i j} B^{2}
\end{aligned}
$$

in Cartesian coordinates. Now we can compute the dilaton $\rho$. The condition (3.21) yields

$$
-\frac{2}{\rho} \partial_{\mu} \rho=T_{\mu \nu \dot{\alpha}} E^{\dot{\alpha} \nu}=\partial_{\mu} E_{0}^{0} E_{0}{ }^{0}+\partial_{\mu} E_{j}^{i} E_{i}{ }^{j}-\partial_{i} E^{0}{ }_{\mu} E_{0}{ }^{i}-\partial_{j} E_{\mu}^{i} E_{i}{ }^{j},
$$

taking into account the above form for the (co)frame. For the space-like components $\mu=k$, this gives

$$
-\frac{2}{\rho} \partial_{k} \rho=A^{-1} \partial_{k} A+2 B^{-1} \partial_{k} B
$$

\footnotetext{
${ }^{5}$ More precisely, the configurations are static on scales much shorter than the cosmic expansion rate.
} 
so that $\rho$ is determined by

$$
A B^{2} \rho^{2}=c_{1}
$$

where $c_{1}$ is a numerical constant. For the time-like components $\mu=0,(6.9)$ leads to

$$
-\frac{2}{\rho} \partial_{0} \rho=0=\frac{1}{B}\left(r E A^{-1} A^{\prime}-\left(r E^{\prime}+3 E\right)\right)
$$

which is solved by

$$
c_{2} A r^{-3}=E .
$$

In particular, we can set $E=0$ for $c_{2}=0$. Furthermore, one finds for the determinants

$$
\operatorname{det}\left(\gamma_{\mu \nu}\right)=-A^{2} B^{6}, \quad \sqrt{|G|}=\frac{c_{1}^{2}}{A B}
$$

where $E$ drops out. In particular, we observe

$$
\sqrt{|G|} \rho^{-2}=c_{1} B
$$

It is straightforward to check that these relations also imply the divergence constraint (3.43). The components of the effective metric $G_{\mu \nu}=\rho^{2} \gamma_{\mu \nu}$ are then obtained explicitly as

$$
\begin{aligned}
G_{00} & =c_{1}\left(-\frac{A}{B^{2}}+r^{2} \frac{E^{2}}{A B^{2}}\right)=c_{1}\left(-1+c_{2}^{2} r^{-4}\right) \frac{A}{B^{2}}, \\
G_{0 i} & =c_{1} \frac{E}{A B} x^{i}=c_{1} c_{2} \frac{1}{A B} r^{-3} x^{i}, \\
G_{i j} & =c_{1} \frac{1}{A} \delta_{i j} .
\end{aligned}
$$

We will focus on the case $E=0$ (and $S=0$ ) in this paper for simplicity, so that $c_{2}=0$. Then

$$
d s_{G}^{2}=-c_{1} \frac{A}{B^{2}} d t^{2}+c_{1} \frac{1}{A} \delta_{i j} d x^{i} d x^{j} .
$$

Equations of motion and solution. For the spherically invariant frames as above, it is convenient to use the formulation (3.50) of the equations of motion. Using

$$
\begin{aligned}
& \star T^{\dot{0}}=r^{-1} A^{\prime} x^{i} \star\left(d x^{i} \wedge d t\right)=r^{-1} A^{\prime} \sqrt{|G|} G^{i i^{\prime}} G^{00} \varepsilon_{i^{\prime} k l} x^{i} d x^{k} \wedge d x^{l} \\
& \star T^{\dot{k}}=r^{-1} B^{\prime} x^{i} \star\left(d x^{i} \wedge d x^{k}\right)=r^{-1} B^{\prime} \sqrt{|G|} G^{i i^{\prime}} G^{k k^{\prime}} \varepsilon_{i^{\prime} k^{\prime} l} x^{i} d t \wedge d x^{l}
\end{aligned}
$$

in Cartesian coordinates (sum over $i$ is understood), we obtain

$$
\begin{aligned}
d\left(\star \rho^{2} T^{\dot{0}}\right)= & r^{-1} A^{\prime} \rho^{2} \sqrt{|G|} G^{i i^{\prime}} G^{00} \varepsilon_{i^{\prime} k l} d x^{i} \wedge d x^{k} \wedge d x^{l} \\
& +\left(r^{-1} A^{\prime} \rho^{2} \sqrt{|G|} G^{i i^{\prime}} G^{00}\right)^{\prime} \varepsilon_{i^{\prime} k l} x^{i} d r \wedge d x^{k} \wedge d x^{l} \\
d\left(\star \rho^{2} T^{\dot{k}}\right)= & r^{-1} B^{\prime} \rho^{2} \sqrt{|G|} G^{i i^{\prime}} G^{k k^{\prime}} \varepsilon_{i^{\prime} k^{\prime} l} d x^{i} \wedge d x^{l} \wedge d t \\
& +r^{-1}\left(r^{-1} B^{\prime} \rho^{2} \sqrt{|G|} G^{i i^{\prime}} G^{k k^{\prime}}\right)^{\prime} \varepsilon_{i^{\prime} k^{\prime} l} x^{i} x^{j} d x^{j} \wedge d x^{l} \wedge d t
\end{aligned}
$$


where ' denotes radial derivative, and $G^{i j} \equiv A(r) \delta^{i j}$. Assuming $m=0$ for simplicity (as well as $S=0=E$ ) and using $\varepsilon_{i k l} x^{i} r d r \wedge d x^{k} \wedge d x^{l}=2 r^{2} d^{3} x$ and $\varepsilon_{i k l} d x^{i} \wedge d x^{k} \wedge d x^{l}=6 d^{3} x$, the equations of motion for $\dot{\alpha}=0$ reduce to

$$
\begin{aligned}
& 0=2 r^{-1}\left(A^{\prime} \rho^{2} \sqrt{|G|} A G^{00}\right)+\left(A^{\prime} \rho^{2} \sqrt{|G|} A G^{00}\right)^{\prime} \\
& 0=\frac{d}{d r}\left(r^{2} \sqrt{|G|} \rho^{2} A G^{00} A^{\prime}\right) .
\end{aligned}
$$

This gives

$$
\frac{d}{d r}\left(r^{2} B^{-1}\left(A^{-1}\right)^{\prime}\right)=0
$$

On the other hand, the space-like equations for $\dot{\alpha}=1,2,3$ have no solutions unless $B^{\prime}=0$; the same conclusion is reached using the formulation (3.49). Thus assume $B(r)=b_{0}=$ const. Then we obtain

$$
\left(A^{-1}\right)^{\prime}=-\frac{M}{r^{2}}, \quad A^{-1}=1+\frac{M}{r}
$$

for some constant $M$. Here we imposed the asymptotic behavior ${ }^{6}$

$$
A(r) \rightarrow 1 \quad \text { for } \quad r \rightarrow \infty
$$

corresponding to an asymptotically constant frame

$$
E^{\dot{0}}=A(r) d t=\frac{1}{1+\frac{M}{r}} d t, \quad E^{\dot{k}}=b_{0} d x^{k}
$$

i.e. $E^{\dot{0}}{ }_{0}=A(r), E_{k}^{\dot{k}}=b_{0}$. This leads to the following non-trivial metric and dilaton

$$
\begin{aligned}
d s_{G}^{2}=G_{\mu \nu} d x^{\mu} d x^{\nu} & =-\frac{c_{1} b_{0}^{-2}}{\left(1+\frac{M}{r}\right)} d t^{2}+c_{1}\left(1+\frac{M}{r}\right) \sum_{i}\left(d x^{i}\right)^{2} \\
& =-\frac{c_{1} b_{0}^{-2}}{\left(1+\frac{M}{r}\right)} d t^{2}+c_{1}\left(1+\frac{M}{r}\right)\left(d r^{2}+r^{2} d \Omega^{2}\right) \\
\rho^{2} & =c_{1} b_{0}^{-2}\left(1+\frac{M}{r}\right) .
\end{aligned}
$$

This reproduces the linearized Schwarzschild metric (cf. [22]), but it deviates from the full Schwarzschild metric at the non-linear level; this should be expected due to the dilaton. The same result can also be obtained from (3.49) using Gauss' theorem which yields (6.21), or directly from (3.49) using the Christoffel symbols. The apparent singularity of the metric at the origin is a coordinate artifact, and the metric is seen (e.g. using $u=\sqrt{r}$ ) to be regular at the origin. We can compute the associated Ricci tensor $\mathcal{R}_{\mu \nu}$ and the Einstein tensor $\mathcal{G}_{\mu \nu}$ : using the relations

$$
\rho^{2} G_{00}=-\alpha^{2}, \quad \alpha:=c_{1} b_{0}^{-2}
$$

\footnotetext{
${ }^{6}$ Strictly speaking we should introduce another normalization constant to recover the cosmic background frame for $r \rightarrow \infty$, which we drop for simplicity.
} 
we find the following result in spherical coordinates for $c_{1}=1$

$$
\begin{aligned}
\mathcal{R}_{\mu \nu} d x^{\mu} d x^{\mu} & =\frac{M^{2}}{2(M+r)^{2}} d \Omega^{2}+\frac{\alpha M^{2}}{2(M+r)^{4}} d t^{2} \\
\mathcal{G}_{\mu \nu} d x^{\mu} d x^{\mu} & =-\frac{M^{2}}{4 r^{2}(M+r)^{2}} d r^{2}+\frac{M^{2}}{4(M+r)^{2}} d \Omega^{2}+\frac{3 \alpha M^{2}}{4(M+r)^{4}} d t^{2} .
\end{aligned}
$$

The Einstein tensor is non-vanishing, but decays like $M^{2} r^{-4}$ as $r \rightarrow \infty$, reflecting the linearized Schwarzschild geometry. ${ }^{7}$ This is clearly integrable at the origin, so that the solution should be viewed as a vacuum or "remnant" solution, rather than representing the gravitational field of matter at the origin. This is possible because the present gravity theory is richer than GR, and the present solution has a non-trivial dilaton. Indeed (D.5) implies that Ricci-flat solutions can arise (in the limit $m^{2} \rightarrow 0$ ) only if both $\rho$ and $\tilde{\rho}$ vanish or cancel each other. It is therefore not surprising that the present solution differs from the Schwarzschild solution. However, we expect that there are other $\mathrm{SO}(3)$-invariant solutions with different characteristics. In particular, such solutions might contribute to the physics underlying the apparent "dark matter".

Finally, it is interesting to note that the total "apparent mass" is proportional to $M$,

$$
\int_{0}^{\infty} d r 4 \pi r^{2} \mathcal{G}_{00}=\pi M
$$

Effective energy-momentum tensor (3.54) and Einstein equations. The above frame has a simple diagonal structure, similar to the background frame. It is related to the dilaton via

$$
E_{0}^{\dot{0}}=A(r)=\alpha \rho^{-2}
$$

using (6.11) and (6.26). This leads to the following expressions for the frame-valued torsion

$$
\begin{aligned}
& T^{\dot{k}}=0 \\
& T^{\dot{0}}=A^{\prime}(r) d r \wedge d t=-\alpha \rho^{-4} d \rho^{2} \wedge d t=\frac{1}{\left(1+\frac{M}{r}\right)^{2}} \frac{M}{r^{2}} d r \wedge d t,
\end{aligned}
$$

so that the non-vanishing components are

$$
T_{i 0}{ }^{\dot{0}}=-T_{0 i}{ }^{\dot{0}}=-2 \alpha \rho^{-3} \partial_{i} \rho
$$

We note that only the time component $E^{\dot{0}}$ of the frame contributes to the torsion, with asymptotic behavior

$$
\begin{array}{ll}
T^{\dot{0}} \sim \frac{1}{r^{2}} \rightarrow 0, & r \rightarrow \infty \\
T^{\dot{0}} \rightarrow \frac{1}{M} \neq 0, & r \rightarrow 0 .
\end{array}
$$

\footnotetext{
${ }^{7}$ Note that we must set $\alpha=1$ if the frame should reduce to the background frame for $r \rightarrow \infty$.
} 
The energy-momentum tensor of the dilaton (D.7) is

$$
\begin{aligned}
\mathbf{T}_{\mu \nu}[\rho] & =2 \rho^{-2}\left(\partial_{\nu} \rho \partial_{\mu} \rho-\frac{1}{2} G_{\nu \mu} \partial^{\sigma} \rho \partial_{\sigma} \rho\right), \\
\mathbf{T}[\rho] & =-2 \rho^{-2} \partial^{\sigma} \rho \partial_{\sigma} \rho=-2 \rho^{-2} G^{r r} \partial_{r} \rho \partial_{r} \rho, \\
\mathbf{T}_{00}[\rho] & =-G_{00} \rho^{-2} \partial^{\sigma} \rho \partial_{\sigma} \rho=\frac{1}{2} G_{00} \mathbf{T}[\rho] .
\end{aligned}
$$

Hence the contribution of the frame to the energy-momentum tensor (D.10) is

$$
\begin{aligned}
\mathbf{T}_{\mu \nu}\left[E^{\dot{0}}\right] & =\rho^{2}\left(T_{\mu \sigma}{ }^{0} T_{\nu \rho}^{\dot{0}} G^{\rho \sigma}-\frac{1}{4} G_{\nu \mu}\left(T_{\sigma \kappa}{ }^{\dot{0}} T_{\sigma^{\prime} \kappa^{\prime}}{ }^{\dot{ }} G^{\sigma \sigma^{\prime}} G^{\kappa \kappa^{\prime}}\right)\right) \\
& =4 \alpha^{2} \rho^{-4}\left(G^{00}\left(\partial_{\mu} \rho \partial_{\nu} \rho-\frac{1}{2} G_{\nu \mu}\left(\partial_{\sigma} \rho \partial_{\sigma^{\prime}} \rho G^{\sigma \sigma^{\prime}}\right)\right)+\delta_{\mu}^{0} \delta_{\nu}^{0}\left(\partial_{\sigma} \rho \partial_{\sigma^{\prime}} \rho G^{\sigma \sigma^{\prime}}\right)\right) \\
& =2 \alpha^{2} \rho^{-2}\left(G^{00} \mathbf{T}_{\mu \nu}[\rho]-\delta_{\mu}^{0} \delta_{\nu}^{0} \mathbf{T}[\rho]\right) \\
& =-2 \mathbf{T}_{\mu \nu}[\rho]+4 \delta_{\mu}^{0} \delta_{\nu}^{0} \mathbf{T}_{00}[\rho]
\end{aligned}
$$

using (6.26), (6.33) and $\rho=\rho(r)$. Note that the frame and the dilaton contribute the same terms with opposite sign, and the total contribution to the Einstein equations is given by

$$
\mathbf{T}_{\mu \nu}=\mathbf{T}_{\mu \nu}\left[E^{\dot{0}}\right]+\mathbf{T}_{\mu \nu}[\rho]=-\mathbf{T}_{\mu \nu}[\rho]+4 \delta_{\mu}^{0} \delta_{\nu}^{0} \mathbf{T}_{00}[\rho] .
$$

Explicitly, for the above solution with $c_{1}=1$ we obtain

$$
\mathbf{T}[\rho]=-\frac{1}{2} \rho^{-4} G^{r r} \partial_{r} \rho^{2} \partial_{r} \rho^{2}=-\frac{1}{2} \frac{1}{\left(1+\frac{M}{r}\right)^{3}} \frac{M^{2}}{r^{4}}
$$

hence

$$
\begin{aligned}
& \mathbf{T}_{00}=3 \mathbf{T}_{00}[\rho]=\frac{3}{2} G_{00} \mathbf{T}[\rho]=\frac{3}{4} \frac{\alpha M^{2}}{(r+M)^{4}} \\
& \mathbf{T}_{r r}=-\mathbf{T}_{r r}[\rho]=\frac{1}{2} G_{r r} \mathbf{T}[\rho]=-\frac{1}{4} \frac{M^{2}}{(r+M)^{2} r^{2}} \\
& \mathbf{T}_{\vartheta \vartheta}=-\mathbf{T}_{\vartheta \vartheta}[\rho]=-\frac{1}{2} G_{\vartheta \vartheta} \mathbf{T}[\rho]=\frac{1}{4} \frac{M^{2}}{(r+M)^{2}} .
\end{aligned}
$$

This agrees precisely with the above results for the Einstein tensor (6.27), as it should. Note that $\mathbf{T}_{\mu \nu} \sim M^{2} r^{-4}$ as $r \rightarrow \infty$. We will give an explicit solution $Z^{\dot{\alpha}}$ below, which realizes this frame via the Poisson brackets.

\section{Semi-classical reconstruction of the matrices $Z_{\dot{\alpha}}$}

Given a classical solution $E_{\dot{\alpha}}{ }^{\mu}$ of the above equations for the frame, we must finally find a corresponding solution $Z_{\dot{\alpha}}$ of the matrix model such that

$$
\left\{Z_{\dot{\alpha}}, x^{\mu}\right\}=E_{\dot{\alpha}}{ }^{\mu} .
$$


This problem was partially solved in [21] sections 6.3 (point 1) and 9.2, where it was shown that the divergence constraint

$$
\partial_{\mu}\left(\rho_{M} E^{\mu}\right)=0
$$

is satisfied for all $\left\{x_{\mu}, Z^{(1)}\right\}_{0} \equiv \mathcal{A}_{\mu}^{(-)}\left[Z^{(1)}\right]$ modes with $Z^{(1)} \in \mathcal{C}^{1}$ (in the notation of [21], cf. (2.14)), and moreover these modes are complete in $\mathcal{C}^{0} \otimes \mathbb{R}^{4}$ (together with the pure gauge modes). Here ()$_{0}$ denotes the projection to the spin 0 sector $\mathcal{C}^{0}$, i.e. to functions on $\mathcal{M}^{3,1}$. This means there is always some $Z_{\dot{\alpha}} \in \mathcal{C}^{1}$ such that $\left\{Z_{\dot{\alpha}}, x^{\mu}\right\}_{0}=E_{\dot{\alpha}}{ }^{\mu}$ as desired, but this may be accompanied by higher spin contributions $\left\{Z_{\dot{\alpha}}, x^{\mu}\right\}_{2} \in \mathcal{C}^{2}$. Whether or not these higher-spin contributions can be canceled by suitable higher-spin "counterterms" or coordinate re-definitions $\tilde{x}^{\mu}$ is an open question in general.

We shall illustrate this reconstruction explicitly for the spherically invariant solution (6.24), by providing explicit functions $Z^{\dot{\alpha}}$ on the bundle space. In that case it is indeed possible to cancel the higher-spin contributions (for the dominant terms at late times), but this requires an infinite tower of higher-spin modes from the point of view of the cosmic background.

Semi-classical $Z^{\dot{\alpha}}$ for the spherical solution. For the specific solution (6.24), we should accordingly find "potentials" $Z^{\dot{\alpha}}$ - and possibly new coordinates $\tilde{x}^{\mu}$ - such that

$$
\begin{array}{lll}
\left\{Z^{0}, \tilde{x}^{0}\right\}=-\sinh (\eta) f\left(r^{2}\right), & & \left\{Z^{0}, \tilde{x}^{i}\right\} \approx 0 \\
\left\{Z^{i}, \tilde{x}^{0}\right\} \approx 0, & & \left\{Z^{i}, \tilde{x}^{j}\right\} \approx \sinh (\eta) \delta^{i j}
\end{array}
$$

for $f(r)=A^{-1}(r)$. Here we re-inserted a factor $\sinh (\eta)$ to recover the background frame (2.19). The second line suggests to leave the space-like generators $Z^{k}=t^{k}$ and the $x^{i}$ coordinates unchanged. Then $x^{0}$ should not be modified either, in order to preserve $\left\{Z^{i}, x^{0}\right\}=0$. The first ansatz one might try is $Z^{0}=t^{0} g(r)$ for some function $g\left(r^{2}\right)$. This would give

$$
\begin{aligned}
\left\{g\left(r^{2}\right) t^{0}, x^{0}\right\} & =g\left(r^{2}\right)\left\{t^{0}, x^{0}\right\}+t^{0}\left\{g\left(r^{2}\right), x^{0}\right\} \\
& =-\sinh (\eta)\left(g\left(r^{2}\right)+2 g^{\prime}\left(r^{2}\right) p_{0}^{2}\right) \in \mathcal{C}^{0} \oplus \mathcal{C}^{2}
\end{aligned}
$$

using (C.12). However, the component in $\mathcal{C}^{2}$ is not subleading and hence cannot be neglected since $\left|p_{0}\right|^{2}=\left|\frac{p \cdot x}{x^{0}}\right|^{2}=r^{2}$, cf. section C.2. Therefore we consider the more general ansatz

$$
\begin{aligned}
Z^{0} & =t^{0} g(r, \chi), & Z^{k} & =t^{k} \\
\tilde{x}^{0} & =x^{0}, & \tilde{x}^{i} & =x^{i}
\end{aligned}
$$

where $\chi$ is the central generator in the $\mathrm{SO}(3)$-invariant sub-algebra given in (C.13), which allows for higher spin corrections. Then

$$
\begin{aligned}
\left\{t^{0} g\left(r^{2}, \chi\right), x^{0}\right\} & =-\sinh (\eta) g\left(r^{2}, \chi\right)-2 \tilde{R} x^{4} \partial_{r^{2}} g\left(r^{2}, \chi\right) t^{0} p^{0} \\
& =-\sinh (\eta) g\left(r^{2}, \chi\right)-\sinh (\eta) \partial_{r^{2}} g\left(r^{2}, \chi\right) 2\left(p^{0}\right)^{2} \\
& =-\sinh (\eta)\left(1+2\left(r^{2}-\chi\right) \partial_{r^{2}}\right) g\left(r^{2}, \chi\right)
\end{aligned}
$$


so that we need to solve

$$
\left(1+2\left(r^{2}-\chi\right) \partial_{r^{2}}\right) g\left(r^{2}, \chi\right)=f\left(r^{2}\right)
$$

The solution is ${ }^{8}$

$$
g\left(r^{2}, \chi\right)=\frac{1}{p_{0}}\left(a(\chi)+\frac{1}{2} \int_{\chi}^{r^{2}} \frac{f(u)}{\sqrt{u-\chi}} d u\right) .
$$

The term with $a(\chi)$ satisfies the homogeneous equation, hence we can simply drop $a(\chi)$, or use it to impose boundary conditions. For the above solution with

$$
f\left(r^{2}\right)=A^{-1}=1+\frac{M}{r}
$$

(assuming $\sinh (\eta) \approx$ const), the above integral can be evaluated explicitly,

$$
\begin{aligned}
\int_{\chi}^{r^{2}} \frac{1+\frac{M}{\sqrt{u}}}{\sqrt{u-\chi}} d u & =2\left(M \log \left(r+\sqrt{r^{2}-\chi}\right)+\sqrt{r^{2}-\chi}\right)-M \log (\chi) \\
& =2\left(M \log \left(r+p_{0}\right)+p_{0}\right)-M \log \left(r^{2}-p_{0}^{2}\right) \\
& =2 p_{0}+M \log \left(\frac{r+p_{0}}{r-p_{0}}\right) .
\end{aligned}
$$

We recall that $\frac{p_{0}}{r} \approx \frac{\vec{p} \cdot \vec{x}}{|\vec{p}||\vec{x}|}=: \cos \vartheta$ and therefore $-r \leq p_{0} \leq r$ due to (C.10) and (C.8), so that the last term is well-defined up to a mild log-type singularity at antipodal points $\vartheta=0, \pi$ of the internal $S^{2}$ fiber. Thus

$$
\tilde{Z}^{0}=t^{0}+\frac{M}{2 R \tilde{R}} \log \left(\frac{r+p_{0}}{r-p_{0}}\right)=t^{0}+\frac{M}{2 R \tilde{R}}\left(\frac{2 p_{0}}{r}+\frac{2}{3} \frac{p_{0}^{3}}{r^{3}}+\ldots\right)=t^{0} A^{-1}(r)+O\left(\frac{p_{0}^{3}}{r^{3}}\right),
$$

which has indeed the structure in (7.3) up to higher-spin corrections. One can check directly that it satisfies the desired relation

$$
\left\{\tilde{Z}^{0}, x^{0}\right\}=-\sinh (\eta)\left(1+\frac{M}{r}\right)=-\sinh (\eta) A^{-1}(r) .
$$

Finally, note that $\left\{\tilde{Z}^{0}, x^{i}\right\}=p^{0}\left\{g, x^{i}\right\} \ll \sinh (\eta)$ is negligible at late times compared to $\left\{\tilde{Z}^{0}, x^{0}\right\}$, because $p^{0}, r^{2}, \chi$ and $\theta^{i j}$ are all bounded i.e. they do not grow with $\eta$. Therefore the $Z^{\dot{\alpha}}$ indeed reconstruct the frame at late times $\eta \gg 1$.

The above solution certainly makes sense as a formal power series, ${ }^{9}$ but we should require that $\tilde{Z}^{0}$ exists as a function on the bundle space $\mathbb{C} P^{1,2}$. As such, the above solution $\tilde{Z}^{0}$ exhibits a logarithmic singularity for $p_{0}= \pm r$, which arises at the antipodes of the internal $S^{2}$ where $\vec{p} \| \vec{x}$. One of them can be canceled by choosing a suitable

\footnotetext{
${ }^{8}$ Here $p^{0}= \pm \sqrt{r^{2}-\chi}$ (C.16) is understood. The explicit $\frac{1}{p_{0}}$ cancels in $\tilde{Z}^{0}$, so that the sign ambiguity drops out. Note that $p^{0}$ has a clean definition as $\mathfrak{s u}(4,2)$ generator at the matrix level, hence this is not a problem.

${ }^{9} \mathrm{Cf}$. the black hole solutions in Vasiliev higher spin theory [33, 34].
} 
$a(\chi)$ in (7.6), but not simultaneously for both singularities. However, this singularity is integrable. This strongly suggests that the solution is acceptable, since the underlying framework of quantized symplectic spaces is based on the relation $\operatorname{End}\left(\mathcal{H}_{n}\right) \sim L^{2}\left(\mathbb{C} P^{1,2}\right)$ between Hilbert-Schmid operators and square-integrable functions on $\mathbb{C} P^{1,2}$. At the operator level, the internal $S^{2}$ is actually a fuzzy sphere [18] and $p^{0}$ arises from a $\mathfrak{s u}(4,2)$ generator $P^{0}$, where the semi-classical bound (C.8) is replaced by a strict operator bound. All this strongly suggests that there should be a well-defined underlying matrix solution, which should be studied in more detail elsewhere.

The situation at $r=0$ is more intricate. Then the location of the singularity on $S^{2}$ becomes inconsistent, which signals a more serious singularity. This suggests that some extra structure is present at the origin. On the other hand, the energy-momentum tensor (6.37) is integrable at the origin. This suggests that the solution should be viewed as a purely geometric matrix configuration with a "quantum" or matrix singularity at the origin. Such a matrix solution might be considered as a "remnant", which is precisely the type of structure where the underlying matrix framework may provide unique new insights.

\section{Discussion}

We have elaborated the non-linear dynamics of the effective gravity sector which emerges from the IKKT or IIB matrix model on a certain type of covariant quantum space. This should be viewed as a candidate for a modified gravity theory. The most convenient description seems to be in terms of the frame $E^{\dot{\alpha}}$, for which we find a simple covariant equation of motion. This captures the equation of motion derived previously for the torsion, avoiding the use of the Weitzenböck connection.

However, there are important differences to the frame formulation of general relativity. The main difference is that the local Lorentz invariance of the frame bundle is broken, and the frame contains more information than just the metric. All frames which arise in the model have the form $E^{\dot{\alpha}}=\left\{Z^{\dot{\alpha}},.\right\}$, which entails a divergence constraint. This is consistent with the fact that diffeomorphism invariance is reduced to a type of volume-preserving diffeomorphisms, which reflects the invariant symplectic volume form on the underlying quantum space. Any such frame leads to a rank 3 tensor which is identified with the torsion of the Weitzenböck connection. The trace of this tensor is related to the dilaton, and the antisymmetric part reduces on-shell to a scalar field identified as axion. These two scalar fields satisfy second-order equations, sourced by the torsion.

Furthermore, we find a simple spherically invariant static solution for the equations of motion, which is localized at some point in space. This solution reproduces the linearized Schwarzschild solution, but deviates from the full Schwarzschild solution at higher order. In particular, there is no horizon, and there is no singularity at the origin, more precisely the singularity is mild and integrable. The effective energy-momentum tensor is smoothly distributed, and can be attributed to the dilaton as well as the time component of the frame field. Therefore this solution is not associated to matter, but should be viewed as a vacuum or perhaps as "remnant" solution, with no counterpart in general relativity. Hence this gravity theory is richer than general relativity, which is potentially very interesting. 
The extra degrees of freedom of the present theory comprise not only the dilaton and axion fields, but also a tower of higher-spin fields, reminiscent of Vasiliev theory. This leads to many open questions. The equations derived for the frame should be viewed as equations for higher-spin valued frames, and it is not evident if the higher-spin contributions to the frame can always be eliminated to reproduce some given metric. For the present solution, we show that this is indeed the case. However, in general the problem of reconstructing frames and the role of the higher-spin fields remains to be clarified.

It is tempting to speculate about the feasibility of the present framework as a physical theory. From this point of view, the deviation from Ricci-flatness is perhaps the most interesting - and challenging - feature: on the one hand it opens up the possibility for geometric explanations of the open problems such as dark energy and dark matter. On the other hand, it remains to be seen if the theory can meet the precision tests of gravity. In particular, the analog of the full Schwarzschild solution arising from matter at the origin is still to be found. It may also be that a proper description of gravity in the presence of matter requires the inclusion of quantum effects, such as an induced Einstein-Hilbert action as discussed previously $[5,14,35]$. In either case, the tools developed in this paper should provide a useful basis for further work towards a more complete understanding of gravity in this remarkable model.

\section{Acknowledgments}

We would especially like to thank Sergio Hörtner for collaboration on this project. HS would also like to thank Yuhma Asano for useful discussions and a related collaboration. The work of HS was supported by the Austrian Science Fund (FWF) grant P32086.

\section{A Conventions and useful formulas}

Levi-Civita symbol. The Levi-Civita symbols will be used with the following convention

$$
\varepsilon_{0123}=1=-\varepsilon^{0123}
$$

so that

$$
\varepsilon_{\nu \rho \sigma \mu} G^{\nu \nu^{\prime}} G^{\sigma \sigma^{\prime}} G^{\mu \mu^{\prime}} G^{\rho \rho^{\prime}}=\left|G^{\mu \nu}\right| \varepsilon^{\nu \rho^{\prime} \sigma \mu^{\prime}}
$$

Contraction of contorsion. We will also need the following contraction formulas which were obtained in (7.54) and (7.55) of [1]

$$
\begin{aligned}
K^{\sigma \rho \mu} K_{\rho \sigma \nu} & =\frac{1}{4}\left(2 T^{\mu \sigma \rho}\left(T_{\nu \rho \sigma}+T_{\nu \sigma \rho}\right)-T^{\rho \sigma \mu} T_{\rho \sigma \nu}\right) \\
K_{\sigma}{ }^{\rho \mu} K_{\rho \mu}{ }^{\sigma}{ }_{\mu} & =\frac{1}{4} T^{\mu \sigma \rho}\left(2 T_{\mu \rho \sigma}+T_{\mu \sigma \rho}\right) .
\end{aligned}
$$


Dilaton and densities. We elaborate the relation between the dilaton $\rho$ (which is a scalar function) and the metric and symplectic densities. To this end, recall that $\rho$ is defined by $\rho^{4}=|\gamma|^{-1} \rho_{M}^{2}=\rho^{8}|G|^{-1} \rho_{M}^{2}$, hence

$$
\rho^{-2}=\sqrt{|G|}^{-1} \rho_{M} .
$$

Here $\rho_{M}$ is the $\mathrm{SO}(4,1)$-invariant volume (density) on $H^{4}$, which is related to the dilaton $\bar{\rho}$ of the cosmic background via the analogous relation

$$
(\bar{\rho})^{-2}=\sqrt{|\bar{G}|}^{-1} \rho_{M}=\sinh ^{-3}(\eta)
$$

cf. (7.23) in [1]. Taking the ratio gives

$$
\frac{\bar{\rho}^{2}}{\rho^{2}}=\frac{\sqrt{|\bar{G}|}}{\sqrt{|G|}}
$$

hence

$$
\sqrt{|G|} \rho^{-2}=\sqrt{|\bar{G}|} \bar{\rho}^{-2}=\rho_{M}\left(=\sinh ^{-1}(\eta)\right)
$$

where the last equality holds only in Cartesian coordinates.

\section{B Divergence constraint and antisymmetric torsion}

\section{B.1 Divergence constraint}

We start with the following identity

$$
\begin{aligned}
T_{\mu \nu}{ }^{\nu}=T_{\mu \nu \dot{\alpha}} E^{\dot{\alpha} \nu} & =\left(\partial_{\mu} E^{\dot{\alpha}}{ }_{\nu}-\partial_{\nu} E^{\dot{\alpha}}{ }_{\mu}\right) E_{\dot{\alpha}}{ }^{\nu} \\
& =\mathcal{E}_{\dot{\alpha}}{ }^{\nu} \partial_{\mu} \mathcal{E}^{\dot{\alpha}}{ }_{\nu}+\mathcal{E}^{\dot{\alpha}}{ }_{\mu} \partial_{\nu} \mathcal{E}_{\dot{\alpha}}{ }^{\nu}-\frac{3}{\rho} \partial_{\mu} \rho
\end{aligned}
$$

where $\mathcal{E}$ is the effective frame (3.19). Together with the relation $-\frac{2}{\rho} \partial_{\mu} \rho=T_{\mu \nu}{ }^{\nu}$ (3.21) for the frame in the matrix model (in the asymptotic regime), we obtain

$$
-\frac{2}{\rho} \partial_{\mu} \rho=\mathcal{E}_{\dot{\alpha}}{ }^{\nu} \partial_{\mu} \mathcal{E}^{\dot{\alpha}}{ }_{\nu}+\mathcal{E}^{\dot{\alpha}}{ }_{\mu} \partial_{\nu} \mathcal{E}_{\dot{\alpha}}{ }^{\nu}-\frac{3}{\rho} \partial_{\mu} \rho .
$$

The first term on the r.h.s. can be rewritten as

$$
\partial_{\mu} \ln (\sqrt{|G|})=\operatorname{det}\left(\mathcal{E}^{\dot{\alpha}}{ }_{\nu}\right)^{-1} \partial_{\mu} \operatorname{det}\left(\mathcal{E}^{\dot{\alpha}}{ }_{\nu}\right)=\mathcal{E}_{\dot{\alpha}}{ }^{\nu} \partial_{\mu} \mathcal{E}^{\dot{\alpha}}{ }_{\nu}
$$

so that

$$
\begin{aligned}
0 & =\partial_{\mu} \ln \sqrt{|G|}+\mathcal{E}_{\mu}^{\dot{\alpha}} \partial_{\nu} \mathcal{E}_{\dot{\alpha}}{ }^{\nu}-\frac{1}{\rho} \partial_{\mu} \rho \\
& =\frac{1}{\sqrt{|G|}} \partial_{\mu} \sqrt{|G|}+\rho^{2} E^{\dot{\alpha}}{ }_{\mu} \partial_{\nu}\left(\rho^{-2} E_{\dot{\alpha}}{ }^{\nu}\right) \\
& =\frac{\rho^{2}}{\sqrt{|G|}} E^{\dot{\alpha}}{ }_{\mu} \partial_{\nu}\left(\sqrt{|G|} \rho^{-2} E_{\dot{\alpha}}{ }^{\nu}\right)
\end{aligned}
$$


which ${ }^{10}$ results in the divergence constraint (3.43),

$$
\partial_{\nu}\left(\sqrt{|G|} \rho^{-2} E_{\dot{\alpha}}{ }^{\nu}\right)=0=\nabla_{\nu}^{(G)}\left(\rho^{-2} E_{\dot{\alpha}}^{\nu}\right)
$$

This means that the $\rho^{-2} E_{\dot{\alpha}}{ }^{\mu}$ are volume-preserving vector fields. It can be viewed as a gauge-fixing relation analogous to a Lorentz gauge condition, which reflects the fact that there is no manifest local Lorentz invariance in the present framework.

The divergence constraint can also be formulated in terms of differential forms. Because of $E_{\dot{\alpha}}{ }^{\nu}=\rho^{2} G^{\nu \mu} E_{\dot{\alpha} \mu}$, we find, using the Hodge star operation with respect to $G$,

$$
d\left(* E_{\dot{\alpha}}\right)=0
$$

Alternative derivation. The origin of the divergence constraint (3.43) from the Jacobi identity can be seen as follows:

$$
\begin{aligned}
E_{\dot{\alpha}}{ }^{\mu} & \sim-\theta^{\mu \nu} \partial_{\nu} Z_{\dot{\alpha}} \\
\partial_{\mu}\left(\rho_{M} E_{\dot{\alpha}}{ }^{\mu}\right) & \sim-\rho_{M} \theta^{\mu \nu} \partial_{\mu} \partial_{\nu} Z_{\dot{\alpha}}=0
\end{aligned}
$$

using $\partial_{\mu}\left(\rho_{M} \theta^{\mu \nu}\right)=0$, which holds in the asymptotic regime. Together with (A.8), we recover

$$
\partial_{\mu}\left(\sqrt{|G|} \rho^{-2} E_{\dot{\alpha}}{ }^{\mu}\right)=0
$$

Yet another derivation is obtained using (6.24) in [23], which implies that $E_{\dot{\alpha}}{ }^{\mu}=\left\{Z_{\dot{\alpha}}, x^{\mu}\right\}_{0}$ for $Z_{\dot{\alpha}} \in \mathcal{C}^{1}$ satisfies

$$
\begin{aligned}
0 & =\bar{\nabla}_{\nu}\left(\sinh ^{-3}(\eta) E_{\dot{\alpha}}{ }^{\mu}\right)=\frac{1}{\sqrt{|\bar{G}|}} \partial_{\nu}\left(\sinh ^{-3}(\eta) \sqrt{|\bar{G}|} E_{\dot{\alpha}}{ }^{\nu}\right) \\
& =\frac{1}{\sqrt{|\bar{G}|}} \partial_{\nu}\left(\sinh ^{-1}(\eta) E_{\dot{\alpha}}{ }^{\nu}\right)=\frac{1}{\sqrt{|\bar{G}|}} \partial_{\nu}\left(\sqrt{|G|} \rho^{-2} E_{\dot{\alpha}}{ }^{\nu}\right)
\end{aligned}
$$

where $\bar{G}$ is the effective metric of the FLRW solution $\mathcal{M}^{3,1}$, and $x^{\mu}$ are Cartesian coordinates.

Divergence constraint and diffeomorphisms. As a consistency check, we verify that the divergence constraint is preserved by the diffeomorphisms which arise from gauge transformations $\{\Lambda,$.$\} in the matrix model. Indeed, the frame transforms under a diffeomor-$ phism generated by the vector field $\xi^{\mu}=\left\{\Lambda, x^{\mu}\right\}$ as

$$
\delta_{\Lambda} E_{\dot{\alpha}}{ }^{\mu}=\mathcal{L}_{\xi} E_{\dot{\alpha}}{ }^{\mu}=\xi^{\nu} \partial_{\nu} E_{\dot{\alpha}}{ }^{\mu}-E_{\dot{\alpha}}{ }^{\nu} \partial_{\nu} \xi^{\mu}
$$

\footnotetext{
${ }^{10}$ This was already shown in (7.18) in [1]
} 
so that the divergence constraint transforms as

$$
\begin{aligned}
\delta_{\Lambda} \partial_{\mu}\left(\sqrt{|G|} \rho^{-2} E_{\dot{\alpha}}{ }^{\mu}\right)= & \partial_{\mu}\left(\sqrt{|G|} \rho^{-2} \xi^{\nu} \partial_{\nu} E_{\dot{\alpha}}{ }^{\mu}\right)-\partial_{\mu}\left(\sqrt{|G|} \rho^{-2} E_{\dot{\alpha}}{ }^{\nu} \partial_{\nu} \xi^{\mu}\right) \\
= & \partial_{\mu} \partial_{\nu}\left(\sqrt{|G|} \rho^{-2} \xi^{\nu} E_{\dot{\alpha}}{ }^{\mu}\right)-\partial_{\mu} \partial_{\nu}\left(\sqrt{|G|} \rho^{-2} E_{\dot{\alpha}}{ }^{\nu} \xi^{\mu}\right) \\
& -\partial_{\mu}\left(\partial_{\nu}\left(\sqrt{|G|} \rho^{-2} \xi^{\nu}\right) E_{\dot{\alpha}}{ }^{\mu}\right) \\
= & -\partial_{\mu}\left(\partial_{\nu}\left(\sqrt{|G|} \rho^{-2} \xi^{\nu}\right) E_{\dot{\alpha}}{ }^{\mu}\right)
\end{aligned}
$$

using (B.5). This reduces precisely to the constraint for the diffeomorphisms (7.3) in [1]

$$
\begin{aligned}
\sqrt{|G|} \nabla_{\nu}^{(G)}\left(\rho^{-2} \xi^{\nu}\right) & =\partial_{\nu}\left(\sqrt{|G|} \rho^{-2} \xi^{\nu}\right)=\partial_{\nu}\left(\rho_{M} \xi^{\nu}\right)=\partial_{\nu}\left(\sqrt{|\bar{G}|} \bar{\rho}^{-2} \xi^{\nu}\right) \\
& =\sqrt{|\bar{G}|} \nabla_{\nu}^{(\bar{G})}\left(\bar{\rho}^{-2} \xi^{\nu}\right)=\sqrt{|\bar{G}|} \nabla_{\nu}^{(\bar{G})}\left(\beta^{3} \xi^{\nu}\right)=0
\end{aligned}
$$

noting that $\sqrt{|G|} \rho^{-2}=\rho_{M}$, where $\bar{G}$ is the cosmic background metric and $\beta^{3}=\sinh ^{-3} \eta=$ $\bar{\rho}^{-2}$. Therefore the divergence constraint is consistent with the volume-preserving diffeos arising in the model. One can also check that it is compatible with the e.o.m. for the frame (3.51).

\section{B.2 The totally antisymmetric torsion $T^{(\mathrm{AS})}$}

The totally antisymmetric torsion is defined by (3.26)

$$
T_{\rho \mu}^{(\mathrm{AS}) \nu}=T_{\rho \mu}^{\nu}+T_{\mu}^{\nu}{ }_{\rho}+T_{\rho \mu}{ }^{\nu} .
$$

Then $G_{\nu \nu^{\prime}} T^{(\mathrm{AS}) \nu^{\prime}}{ }_{\rho \mu}$ is totally antisymmetric in the indices $\nu, \rho, \mu$ and can naturally be interpreted as a 3 -form $T^{(\mathrm{AS})}$. It is related by the Hodge star $\star$ corresponding to $G_{\mu \nu}$ to a 1-form $T_{\sigma} d x^{\sigma}$ via

$$
T^{(\mathrm{AS})}:=\frac{1}{6} G_{\nu \nu^{\prime}} T_{\rho \mu}^{(\mathrm{AS}) \nu^{\prime}} d x^{\nu} \wedge d x^{\rho} \wedge d x^{\mu}=\star\left(T_{\sigma} d x^{\sigma}\right) .
$$

In coordinates, this amounts to

$$
\begin{aligned}
T_{\rho \mu}^{(\mathrm{AS}) \nu} & =-\sqrt{|G|} G^{\nu \nu^{\prime}} \varepsilon_{\nu^{\prime} \rho \mu \sigma} G^{\sigma \sigma^{\prime}} T_{\sigma^{\prime}}, \\
\varepsilon^{\nu^{\prime} \rho \mu \kappa} G_{\nu^{\prime} \nu} T_{\rho \mu}^{(\mathrm{AS}) \nu} & =6 \sqrt{|G|} G^{\kappa \sigma^{\prime}} T_{\sigma^{\prime}}
\end{aligned}
$$

using the conventions (A.1). This implies

$$
\begin{aligned}
T_{\rho \mu}^{(\mathrm{AS}) \sigma} T_{\sigma \nu}^{(\mathrm{AS}){ }_{\nu}} & =|G| G^{\sigma \sigma^{\prime}} \varepsilon_{\sigma^{\prime} \rho \mu \kappa} G^{\rho \rho^{\prime}} \varepsilon_{\sigma \rho^{\prime} \nu \eta} G^{\kappa \kappa^{\prime}} T_{\kappa^{\prime}} G^{\eta \eta^{\prime}} T_{\eta^{\prime}} \\
& =\varepsilon^{\sigma \rho \mu^{\prime} \kappa} \varepsilon_{\sigma \rho \nu \eta} G_{\mu^{\prime} \mu} T_{\kappa} G^{\eta \eta^{\prime}} T_{\eta^{\prime}} \\
& =2\left(T_{\nu} T_{\mu}-G_{\nu \mu} G^{\kappa \eta} T_{\kappa} T_{\eta}\right)
\end{aligned}
$$

using (A.2), and the contraction gives

$$
T_{\rho \mu}^{(\mathrm{AS}) \sigma} T_{\sigma \nu}^{(\mathrm{AS}){ }_{\nu}} G^{\mu \nu}=-6 T_{\nu} T_{\mu} G^{\mu \nu} .
$$


We also note the following identity

$$
T_{\rho \mu}^{(\mathrm{AS}) \sigma} T_{\sigma \nu}^{(\mathrm{AS}) \rho}=-2\left(T_{\mu}{ }^{\sigma}{ }_{\rho} T_{\sigma \nu}^{\rho}+T_{\nu}{ }_{\rho}^{\sigma} T_{\sigma \mu}^{\rho}\right)+2\left(T_{\mu}{ }_{\rho}{ }_{\rho} T_{\nu \sigma}{ }^{\rho}-T_{\mu}{ }^{\sigma}{ }_{\rho} T_{\nu \sigma}{ }^{\rho}\right)+T_{\rho \mu}^{\sigma} T_{\sigma}{ }^{\rho}{ }_{\nu} .
$$

Contracting this with $G^{\mu \nu}$ gives

$$
\begin{aligned}
T_{\rho \mu}^{(\mathrm{AS}) \sigma} T_{\sigma}(\mathrm{AS}){ }_{\nu} G^{\mu \nu} & =3\left(T_{\mu}{ }^{\sigma} T_{\nu \sigma}{ }^{\rho} G^{\mu \nu}-2 T_{\mu \rho}^{\sigma} T_{\sigma}{ }^{\rho}{ }_{\nu} G^{\mu \nu}\right) \\
T_{\mu \rho}^{\sigma} T_{\sigma}{ }_{\nu}{ }_{\nu} G^{\mu \nu} & =\frac{1}{2} T_{\mu}{ }^{\sigma} T_{\nu \sigma}{ }^{\rho} G^{\mu \nu}-\frac{1}{6} T_{\rho \mu}^{(\mathrm{AS}) \sigma} T_{\sigma}^{(\mathrm{AS})}{ }_{\nu} G^{\mu \nu} \\
& =\frac{1}{2} T_{\mu}{ }^{\sigma}{ }_{\rho} T_{\nu \sigma}{ }^{\rho} G^{\mu \nu}+T_{\nu} T_{\mu} G^{\mu \nu} .
\end{aligned}
$$

We will also need the formulas

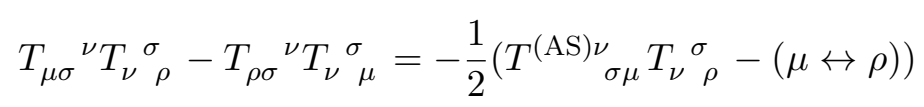

and

$$
2 K_{\nu \rho}{ }^{\sigma} T_{\sigma \mu}{ }^{\nu}+2 K_{\nu \mu}{ }^{\sigma} T_{\rho \sigma}{ }^{\nu}=-\frac{1}{2}\left(T_{\sigma \mu}^{(\mathrm{AS}) \nu} T_{\nu}{ }^{\sigma} \rho-(\mu \leftrightarrow \rho)\right)
$$

The divergence of $\boldsymbol{T}_{\boldsymbol{\mu}}$. The divergence of $T_{\sigma}$ satisfies the identity

$$
\begin{aligned}
G^{\kappa \sigma} & \nabla_{\kappa}^{(G)} T_{\sigma} \\
& =\frac{1}{6} \sqrt{|G|}^{-1} \varepsilon^{\nu^{\prime} \rho \mu \kappa} G_{\nu^{\prime} \nu} \nabla_{\kappa}^{(G)} T_{\rho \mu}^{(\mathrm{AS}) \nu}{ }_{\rho \mu} \\
& =\frac{1}{2} \sqrt{|G|}^{-1} \varepsilon^{\nu \rho \mu^{\prime} \kappa} G_{\mu^{\prime} \mu} \nabla_{\kappa}^{(G)} T_{\nu \rho}{ }^{\mu} \\
& =\frac{1}{2} \sqrt{|G|}^{-1} \varepsilon^{\nu \rho \mu^{\prime} \kappa} G_{\mu^{\prime} \mu} \nabla_{\kappa}^{(G)}\left(\left(\nabla_{\nu}^{(G)} E^{\dot{\alpha}}{ }_{\rho}-\nabla_{\rho}^{(G)} E^{\dot{\alpha}}{ }_{\nu}\right) E_{\dot{\alpha}}{ }^{\mu}\right) \\
& =\frac{1}{2} \sqrt{|G|}^{-1} \varepsilon^{\nu \rho \mu^{\prime} \kappa} G_{\mu^{\prime} \mu}\left(\frac{1}{2} \mathcal{R}_{\kappa \nu}{ }^{\sigma}{ }_{\rho} E^{\dot{\alpha}}{ }_{\sigma}-\frac{1}{2} \mathcal{R}_{\kappa \rho}{ }^{\sigma}{ }_{\nu} E^{\dot{\alpha}}{ }_{\sigma}+\left(\nabla_{\nu}^{(G)} E_{\rho}^{\dot{\alpha}}{ }_{\rho}-\nabla_{\rho}^{(G)} E^{\dot{\alpha}}{ }_{\nu}\right) \nabla_{\kappa}^{(G)} E_{\dot{\alpha}}{ }^{\mu}\right) \\
& =\frac{1}{2} \sqrt{|G|}^{-1} \varepsilon^{\nu \rho \mu \kappa}\left(\left(\nabla_{\nu}^{(G)} E^{\dot{\alpha}}{ }_{\rho}-\nabla_{\rho}^{(G)} E^{\dot{\alpha}}{ }_{\nu}\right) \nabla_{\kappa}^{(G)}\left(\rho^{2} E_{\dot{\alpha} \mu}\right)\right) \\
& =\frac{1}{4} \rho^{2} \sqrt{|G|}^{-1} \varepsilon^{\nu \rho \mu \kappa} T_{\nu \rho}{ }^{\dot{\alpha}} T_{\kappa \mu \dot{\alpha}}+\frac{1}{2}\left(\partial_{\kappa} \rho^{2}\right) \sqrt{|G|}^{-1} \varepsilon^{\nu \rho \mu \kappa} T_{\nu \rho \mu} \\
& =\frac{1}{4} \rho^{2} \sqrt{|G|}^{-1} \varepsilon^{\nu \rho \mu \kappa} T_{\nu \rho}{ }^{\dot{\alpha}} T_{\kappa \mu \dot{\alpha}}+\frac{1}{6} \rho^{-2} \partial_{\kappa} \rho^{2} \sqrt{|G|}^{-1} \varepsilon^{\nu \rho \mu \kappa} G_{\mu \mu^{\prime}} T^{(\mathrm{AS})}{ }_{\nu \rho}{ }^{\prime \prime} \\
& =\frac{1}{4} \rho^{2} \sqrt{|G|}^{-1} \varepsilon^{\nu \rho \mu \kappa} T_{\nu \rho}{ }^{\dot{\alpha}} T_{\kappa \mu \dot{\alpha}}+2 \rho^{-1} \partial_{\kappa} \rho G^{\kappa \kappa^{\prime}} T_{\kappa^{\prime}}
\end{aligned}
$$

which follows from (B.15) using the algebraic Bianchi identity for the Riemann tensor. Multiplied by $\rho^{-2}$ this leads to the identity

$$
\tilde{\rho} \varepsilon^{\nu \rho \mu \kappa} T_{\nu \rho}{ }^{\dot{\alpha}} T_{\kappa \mu \dot{\alpha}}=4 \sqrt{|G|} \tilde{\rho} G^{\mu \nu} \nabla_{\mu}^{(G)}\left(\rho^{-2} T_{\nu}\right)
$$


and using the e.o.m. (3.36) for $T_{\mu}$ this implies

$$
\tilde{\rho} \varepsilon^{\nu \rho \mu \kappa} T_{\nu \rho}^{\dot{\alpha}} T_{\kappa \mu \dot{\alpha}}=4 \sqrt{|G|} \tilde{\rho} G^{\mu \nu} \nabla_{\mu}^{(G)}\left(\rho^{-4} \partial_{\nu} \tilde{\rho}\right) .
$$

As a by-product, we obtain the following interesting identity

$$
S_{\tilde{E}}=-\int d^{4} x \tilde{\rho} \varepsilon^{\nu \rho \mu \kappa} T_{\nu \rho}{ }^{\dot{\alpha}} T_{\kappa \mu \dot{\alpha}}=4 \int d^{4} x \sqrt{|G|} \rho^{-4} G^{\mu \nu} \partial_{\mu} \tilde{\rho} \partial_{\nu} \tilde{\rho}=4 S_{\tilde{\rho}} .
$$

Since the first term has the structure $\int \tilde{\rho} d E \wedge d E$, this suggests to view $\tilde{\rho}$ as an axion associated to the frame.

\section{6-dimensional configuration space and constraints}

\section{C.1 General setup}

We want to describe the 6-dimensional symplectic space $\mathcal{M}^{(6)}(2.6)$ underlying the cosmic background solution more explicitly. It is described through the 8 functions $x^{\mu}$ and $t^{\mu}$ subject to the constraints (2.13), which transform covariantly under the global $\operatorname{SO}(3,1)$ isometry group of the $k=-1$ FLRW spacetime $\mathcal{M}^{3,1}$. To exhibit the $S^{2}$ bundle structure over $\mathcal{M}^{3,1}$, it is useful to consider $\mathcal{M}^{(6)}$ as Cartesian product ${ }^{11}$

$$
\mathcal{M}^{(6)} \cong \mathbb{R}_{x}^{3} \times \mathbb{R}_{p}^{3}
$$

described by the space-like Cartesian coordinates $x^{i}, p^{j}$, while $x^{0}$ and $p^{0}$ are determined by the constraints. This is most transparent in terms of the re-scaled generators

$$
p^{\alpha}=\tilde{R} R t^{\alpha}
$$

with Poisson brackets

$$
\left\{p^{\mu}, x^{\nu}\right\}=\tilde{R} x^{4} \eta^{\mu \nu}, \quad x^{4}=\sqrt{x_{\mu} x^{\mu}+R^{2}} .
$$

Then the constraints (2.13) take the form

$$
\begin{aligned}
p_{0} x^{0} & =p_{k} x^{k}, \\
-\left(p_{0}\right)^{2}+p_{i} p_{i} & =\left(x_{0}\right)^{2}-r^{2}
\end{aligned}
$$

(sum over $i$ is understood) where

$$
r^{2}=x_{i} x_{i}
$$

The constraints can be written as

$$
\begin{aligned}
& \left(p_{0}+x_{0}\right)^{2}=p_{i} p_{i}+r^{2}+2 p_{k} x^{k}=|\vec{x}+\vec{p}|^{2} \\
& \left(p_{0}-x_{0}\right)^{2}=p_{i} p_{i}+r^{2}-2 p_{k} x^{k}=|\vec{x}-\vec{p}|^{2}
\end{aligned}
$$

\footnotetext{
${ }^{11}$ This description misses the double cover structure of $\mathcal{M}^{(6)} \cong \mathbb{C} P^{1,2}[14]$, but it captures the structure after the Big Bounce.
} 
which is solved by

$$
\begin{aligned}
& x_{0}=\frac{1}{2}(|\vec{x}+\vec{p}|+|\vec{x}-\vec{p}|)>0 \\
& p_{0}=\frac{1}{2}(|\vec{x}+\vec{p}|-|\vec{x}-\vec{p}|)
\end{aligned}
$$

which reproduces the cosmic background (after the Big Bounce). Note that $p_{0}$ can take either sign, consistent with (C.4), and the triangle inequality applied to (C.7) implies the bound

$$
\left|p_{0}\right| \leq|\vec{x}|
$$

For a reference point $\xi \in \mathcal{M}^{3,1}$ with $\vec{x}=0$, this gives

$$
x_{0}=|p|, \quad p_{0}=0 .
$$

More generally in a local region near $\xi$ with $|p|=x_{0} \gg r$, we have

$$
\begin{aligned}
& x^{0}=\frac{1}{2}\left(\sqrt{p^{2}+r^{2}+2 p x}+\sqrt{p^{2}+r^{2}-2 p x}\right) \approx|p| \\
& p_{0}=\frac{1}{2}\left(\sqrt{p^{2}+r^{2}+2 p x}-\sqrt{p^{2}+r^{2}-2 p x}\right) \approx \frac{p x}{|p|} \approx \frac{p x}{x_{0}}
\end{aligned}
$$

up to corrections suppressed by $\frac{r}{x^{0}}$. Therefore $\left|x^{0}\right| \approx|p| \approx$ const. is essentially the cosmic time, which completes the spacetime coordinates on $\mathcal{M}^{3,1}$

$$
x^{\mu}=\left(x^{0} \approx|p|, x^{i}\right),
$$

while the $S^{2}$ fiber is described by the two transversal $p^{i}$.

\section{C.2 SO(3)-invariant functions and Poisson brackets}

Consider the subalgebra of $\mathrm{SO}(3)$-invariant functions of $r^{2}, x^{0}, p^{0}$ using the notation (C.2). Here $r^{2}, x^{0}$ are viewed as elements of the algebra $\mathcal{C}^{0}$ describing the spin 0 sector of functions on $\mathcal{M}^{3,1}$, while $p^{0} \in \mathcal{C}^{1}$ is a higher spin generator (since it transforms non-trivially under the local $\mathrm{SO}(3))$. The Poisson brackets are

$$
\begin{aligned}
& \left\{r^{2}, p^{0}\right\}=0 \\
& \left\{r^{2}, x^{0}\right\}=-2 \tilde{R} x^{4} p^{0} \\
& \left\{p^{0}, x^{0}\right\}=-\tilde{R} x^{4} .
\end{aligned}
$$

They span a space of functions in 3 variables. Therefore there must be a central generator, and the symplectic leaves are 2-dimensional. This generator is found to be

$$
\{\chi, .\}=0, \quad \chi:=r^{2}-\left(p^{0}\right)^{2}=x_{0}^{2}-|\vec{p}|^{2}
$$


which is central within the $\mathrm{SO}(3)$-invariant subalgebra. Indeed,

$$
\begin{aligned}
\left\{\chi, r^{2}\right\} & =-2 p^{0}\left\{p^{0}, r^{2}\right\}=0 \\
\left\{\chi, x^{0}\right\} & =\left\{r^{2}, x^{0}\right\}-2 p^{0}\left\{p^{0}, x^{0}\right\} \\
& =-2 \tilde{R} x^{4} p^{0}+2 \tilde{R} x^{4} p^{0}=0 .
\end{aligned}
$$

We also note that (C.8) implies

$$
r^{2} \geq \chi \geq 0
$$

and in particular

$$
p^{0}= \pm \sqrt{r^{2}-\chi}
$$

\section{Geometric energy-momentum tensor}

It was shown in (eq. (5.70) in [1]) that the Ricci tensor in vacuum satisfies the following vacuum equation of motion

$$
\begin{aligned}
\mathcal{R}_{\nu \mu}= & -\frac{1}{2}\left(T_{\rho}{ }^{\delta}{ }_{\mu} T_{\nu \delta}{ }^{\rho}+T_{\rho}{ }_{\nu}{ }_{\nu} T_{\mu \delta}{ }^{\rho}\right)-K_{\delta}{ }^{\rho}{ }_{\nu} K_{\rho \mu}{ }^{\delta}+2 \rho^{-2} \partial_{\nu} \rho \partial_{\mu} \rho \\
& +G_{\nu \mu}\left(\rho^{-2} m^{2}-\frac{1}{2} T_{\nu}{ }^{\sigma}{ }_{\delta} T_{\sigma \rho}{ }^{\nu} G^{\delta \rho}\right) .
\end{aligned}
$$

The first three terms can be rewritten using (A.3) and (B.18) as

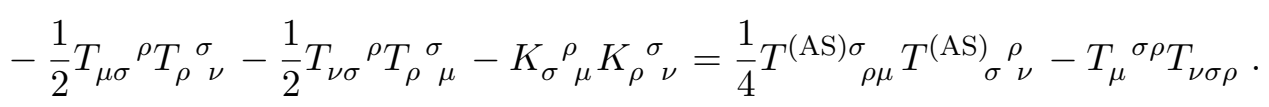

Therefore the on-shell Ricci tensor (D.1) can be written as

$$
\begin{aligned}
\mathcal{R}_{\nu \mu}= & \frac{1}{4} T_{\rho \mu}^{(\mathrm{AS}) \sigma} T_{\sigma \nu}^{(\mathrm{AS}){ }_{\nu}}-T_{\mu \sigma}{ }^{\rho} T_{\nu}{ }_{\rho}{ }_{\rho}+2 \rho^{-2} \partial_{\nu} \rho \partial_{\mu} \rho \\
& +\frac{1}{4} G_{\nu \mu}\left(4 \rho^{-2} m^{2}+T_{\nu \delta}^{\sigma} T_{\sigma}{ }^{\nu}{ }_{\rho} G^{\delta \rho}-\frac{1}{3} T_{\rho \mu}^{(\mathrm{AS}) \sigma} T_{\sigma}^{(\mathrm{AS}){ }_{\nu}{ }_{\nu}} G^{\mu \nu}\right)
\end{aligned}
$$

and

$$
\mathcal{R}=-\frac{1}{12} T_{\rho \mu}^{(\mathrm{AS}) \sigma} T_{\sigma \nu}^{(\mathrm{AS})} G^{\mu \nu}+2 \rho^{-2} \partial_{\mu} \rho \partial^{\mu} \rho+4 \rho^{-2} m^{2}
$$

Using (B.17) and the on-shell relation (3.36) for $T_{\mu}$, we obtain the on-shell relation

$$
\mathcal{R}=\frac{1}{2} \rho^{-4} G^{\mu \nu} \partial_{\mu} \tilde{\rho} \partial_{\nu} \tilde{\rho}+2 \rho^{-2} \partial_{\mu} \rho \partial^{\mu} \rho+4 \rho^{-2} m^{2} .
$$

This implies that solutions with $\mathcal{R}=0$ can arise (in the limit $m^{2} \rightarrow 0$ ) only if both $\rho$ and $\tilde{\rho}$ vanish or cancel each other. 
Effective energy-momentum tensor. We now define the effective energy-momentum (e-m) tensor due to torsion in terms of the Einstein equations (absorbing a factor $8 \pi$ in the e-m tensor), which thus decomposes into different contributions

$$
\begin{aligned}
& \mathbf{T}_{\mu \nu}:=\mathcal{R}_{\nu \mu}-\frac{1}{2} G_{\mu \nu} \mathcal{R}
\end{aligned}
$$

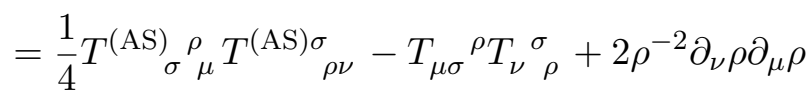

$$
\begin{aligned}
& +\frac{1}{4} G_{\nu \mu}\left(-4 \rho^{-2} m^{2}+T_{\nu \delta}^{\sigma} T_{\sigma}{ }_{\rho}^{\nu} G^{\delta \rho}-\frac{1}{6} T_{\sigma \mu}^{(\mathrm{AS})_{\rho}{ }_{\mu}} T_{\rho \nu}^{(\mathrm{AS}) \sigma} G^{\mu \nu}-4 \rho^{-2} \partial^{\mu} \rho \partial_{\mu} \rho\right) \\
& =\mathbf{T}_{\mu \nu}\left[E^{\dot{\alpha}}\right]+\mathbf{T}_{\mu \nu}[\rho]+\mathbf{T}_{\mu \nu}^{(\mathrm{AS})}[T]-\rho^{-2} m^{2} G_{\nu \mu} .
\end{aligned}
$$

Here the energy-momentum tensor of the dilaton is

$$
\begin{aligned}
\mathbf{T}_{\mu \nu}[\rho] & =2 \rho^{-2}\left(\partial_{\nu} \rho \partial_{\mu} \rho-\frac{1}{2} G_{\nu \mu} \partial^{\sigma} \rho \partial_{\sigma} \rho\right) \\
& =2\left(\partial_{\nu} \sigma \partial_{\mu} \sigma-\frac{1}{2} G_{\nu \mu} \partial^{\kappa} \sigma \partial_{\kappa} \sigma\right), \quad \rho=e^{-\sigma} .
\end{aligned}
$$

For the contribution of $T^{(\mathrm{AS})}$ we can use (B.16) and (B.17), so that

$$
\begin{aligned}
\mathbf{T}_{\mu \nu}^{(\mathrm{AS})}[T] & =\frac{1}{4}\left(T_{\rho \mu}^{(\mathrm{AS}) \sigma} T_{\sigma \nu}^{(\mathrm{AS})}{ }_{\nu}-\frac{1}{6} G_{\nu \mu}\left(T_{\rho \mu}^{(\mathrm{AS}) \sigma} T_{\sigma \nu}^{(\mathrm{AS}){ }_{\nu}} G^{\mu \nu}\right)\right) \\
& =\frac{1}{2}\left(T_{\mu} T_{\nu}-\frac{1}{2} G_{\nu \mu}\left(T^{\rho} T_{\rho}\right)\right) .
\end{aligned}
$$

Using the e.o.m. (3.36) for $T_{\mu}$, this reduces to the e-m tensor of the axion,

$$
\mathbf{T}^{(\mathrm{AS})}{ }_{\mu \nu}[T]=\frac{1}{2} \rho^{-4}\left(\partial_{\mu} \tilde{\rho} \partial_{\nu} \tilde{\rho}-\frac{1}{2} G_{\nu \mu}\left(G^{\sigma \sigma^{\prime}} \partial_{\sigma} \tilde{\rho} \partial_{\sigma^{\prime}} \tilde{\rho}\right)\right)=: \mathbf{T}_{\mu \nu}[\tilde{\rho}]
$$

Finally, the e-m tensor of the frame is

$$
\begin{aligned}
\mathbf{T}_{\mu \nu}\left[E^{\dot{\alpha}}\right] & :=-T_{\mu \sigma}{ }^{\rho} T_{\nu}{ }^{\sigma}{ }+\frac{1}{4} G_{\nu \mu} T^{\sigma}{ }_{\nu \delta} T_{\sigma}{ }_{\rho}^{\nu} G^{\delta \rho} \\
& =-\rho^{2}\left(F_{\mu \sigma}\left[E^{\dot{\alpha}}\right] F_{\nu \rho}\left[E_{\dot{\alpha}}\right] G^{\rho \sigma}-\frac{1}{4} G_{\nu \mu}\left(F_{\rho \kappa}\left[E^{\dot{\alpha}}\right] F_{\sigma \kappa^{\prime}}\left[E_{\dot{\alpha}}\right] G^{\rho \sigma} G^{\kappa \kappa^{\prime}}\right)\right)
\end{aligned}
$$

where

$$
F_{\mu \nu}\left[E^{\dot{\alpha}}\right]:=\partial_{\mu} E_{\nu}^{\dot{\alpha}}-\partial_{\nu} E_{\mu}^{\dot{\alpha}}=T_{\mu \nu}^{\dot{\alpha}}
$$

is the field strength of the frame vector field. Thus $\mathbf{T}_{\mu \nu}\left[E^{\alpha}\right]$ has the structure of a negative Maxwell-like e-m tensor

$$
\mathbf{T}_{\mu \nu}[A]=F_{\mu \rho} F_{\nu \sigma} G^{\rho \sigma}-\frac{1}{4} G_{\mu \nu}\left(F_{\rho \sigma} F^{\rho \sigma}\right), \quad F=d A
$$

from each frame component $E^{\dot{\alpha}}$, with opposite sign for space- and time-like components. Hence it is possible in principle to obtain Ricci-flat solutions, provided the various contributions with different signs cancel. 


\section{E Geometric actions and identities}

\section{E.1 Einstein-Hilbert term from torsion}

We start from the identity (5.61) in [1] and obtain the Ricci scalar as

$$
\begin{aligned}
\mathcal{R}[G] & =2 \nabla_{(G)}^{\mu} \mathcal{K}_{\nu \mu}{ }^{\nu}-\mathcal{K}_{\mu \rho}{ }^{\mu} \mathcal{K}_{\nu \sigma}{ }^{\rho} G^{\nu \sigma}+\mathcal{K}_{\nu \rho}{ }^{\mu} \mathcal{K}_{\mu \sigma}{ }^{\rho} G^{\nu \sigma} \\
& =\mathcal{K}_{\nu \rho}{ }^{\mu} \mathcal{K}_{\mu \sigma}{ }^{\rho} G^{\nu \sigma}-\mathcal{K}_{\mu \rho}{ }^{\mu} \mathcal{K}_{\nu \sigma}{ }^{\rho} G^{\nu \sigma}-2 \nabla_{(G)}^{\mu}\left(\rho^{-1} \partial_{\mu} \rho\right) \\
& =K_{\nu \rho}{ }^{\mu} K_{\mu \sigma}{ }^{\rho} G^{\nu \sigma}+2 \rho^{-2} G^{\mu \nu} \partial_{\mu} \rho \partial_{\nu} \rho-2 \nabla_{(G)}^{\mu}\left(\rho^{-1} \partial_{\mu} \rho\right) \\
& =-\frac{1}{4} T^{\mu}{ }_{\sigma \rho} T_{\mu \sigma^{\prime}}{ }^{\rho} G^{\sigma \sigma^{\prime}}-\frac{1}{2} T^{\mu}{ }_{\sigma \rho} T_{\mu}{ }^{\rho} \sigma^{\prime} G^{\sigma \sigma^{\prime}}+2 \rho^{-2} G^{\mu \nu} \partial_{\mu} \rho \partial_{\nu} \rho-2 \nabla_{(G)}^{\mu}\left(\rho^{-1} \partial_{\mu} \rho\right)
\end{aligned}
$$

using (3.17), the contraction relation (3.21), and (A.4) in the last step. This is an identity, no equations of motion are used. Upon integration, we can rewrite the second term as

$$
\begin{aligned}
-\int d^{4} x \sqrt{|G|} T^{\sigma}{ }_{\mu \rho} T_{\sigma}{ }_{\nu} G^{\mu \nu} & =2 \int d^{4} x \sqrt{|G|} \nabla_{\mu}^{(G)} E_{\sigma}^{\dot{\alpha}} E_{\dot{\alpha}}{ }^{\rho}\left(\nabla_{\sigma^{\prime}}^{(G)} E_{\rho}^{\dot{\beta}}-\nabla_{\rho}^{(G)} E_{\sigma^{\prime}}^{\dot{\beta}}\right) E_{\dot{\beta}}{ }^{\mu} G^{\sigma \sigma^{\prime}} \\
& =4 \int d^{4} x \sqrt{|G|} E_{\dot{\beta}}{ }^{\mu} \nabla_{\mu}^{(G)} E^{\dot{\alpha}}{ }_{\sigma} \nabla_{\sigma^{\prime}}^{(G)} E^{\dot{\beta}}{ }_{\rho} E_{\dot{\alpha}}{ }^{\rho} G^{\sigma \sigma^{\prime}} \\
& =-4 \int d^{4} x \sqrt{|G|} \nabla_{\mu}^{(G)} E^{\dot{\alpha}}{ }_{\sigma} \nabla_{\sigma^{\prime}}^{(G)} E_{\dot{\alpha}}{ }^{\mu} G^{\sigma \sigma^{\prime}} \\
& =4 \int d^{4} x \sqrt{|G|} \nabla_{\sigma^{\prime}}^{(G)} \nabla_{\mu}^{(G)} E^{\dot{\alpha}}{ }_{\sigma} E_{\dot{\alpha}}{ }^{\mu} G^{\sigma \sigma^{\prime}} \\
& =4 \int d^{4} x \sqrt{|G|}\left(\nabla_{\mu}^{(G)} \nabla_{(G)}^{\sigma} E^{\dot{\alpha}}{ }_{\sigma} E_{\dot{\alpha}}{ }^{\mu}+G^{\sigma \sigma^{\prime}} \mathcal{R}_{\sigma^{\prime} \mu ; \sigma \kappa} G^{\kappa \mu}\right) \\
& =4 \int d^{4} x \sqrt{|G|}\left(-\nabla_{\sigma^{\prime}}^{(G)} E^{\dot{\alpha} \sigma} \nabla_{\mu}^{(G)} E_{\dot{\alpha}}{ }^{\mu}+\mathcal{R}\right) \\
& =4 \int d^{4} x \sqrt{|G|}\left(-4 \rho^{-2} \partial_{\mu} \rho \partial^{\mu} \rho+\mathcal{R}\right)
\end{aligned}
$$

using the divergence constraint (3.43). Then (E.1) gives

$$
\int d^{4} x \sqrt{|G|} \mathcal{R}[G]=\int d^{4} x \sqrt{|G|}\left(-\frac{1}{4} T_{\sigma \rho}^{\mu} T_{\mu \sigma^{\prime}}{ }^{\rho} G^{\sigma \sigma^{\prime}}+2\left(-3 \rho^{-2} \partial_{\mu} \rho \partial^{\mu} \rho+\mathcal{R}\right)\right)
$$

hence

$$
\int d^{4} x \sqrt{|G|} \mathcal{R}[G]=\int d^{4} x \sqrt{|G|}\left(\frac{1}{4} T_{\sigma \rho}^{\mu} T_{\mu \sigma^{\prime}}{ }^{\rho} G^{\sigma \sigma^{\prime}}+6 \rho^{-2} G^{\mu \nu} \partial_{\mu} \rho \partial_{\nu} \rho\right) .
$$

This is an identity which applies to any off-shell configuration. Thus all action terms quadratic in the torsion can be written in terms of the Einstein-Hilbert term and the dilaton. Similar identities are used in the teleparallel reformulation of general relativity [28], but the role of the dilaton and the divergence constraint is specific to the present framework. 


\section{E.2 Axion identity}

The identities (E.2), (B.19) and (E.4) can be combined as follows

$$
\begin{aligned}
4 \int d^{4} x \sqrt{|G|}\left(-4 \rho^{-2} \partial_{\mu} \rho \partial^{\mu} \rho+\mathcal{R}\right) & =-\int d^{4} x \sqrt{|G|} T_{\mu \rho}^{\sigma} T_{\sigma}{ }_{\nu} G^{\mu \nu} \\
& =\int d^{4} x \sqrt{|G|}\left(-\frac{1}{2} T_{\mu}{ }^{\sigma}{ }_{\rho} T_{\nu \sigma}{ }^{\rho} G^{\mu \nu}-T_{\nu} T_{\mu} G^{\mu \nu}\right) \\
& =\int d^{4} x \sqrt{|G|}\left(-2 \mathcal{R}[G]+12 \rho^{-2} G^{\mu \nu} \partial_{\mu} \rho \partial_{\nu} \rho-T_{\nu} T_{\mu} G^{\mu \nu}\right) .
\end{aligned}
$$

Therefore all configurations of the matrix model satisfy

$$
0=\int d^{4} x \sqrt{|G|}\left(-3 \mathcal{R}+14 \rho^{-2} G^{\mu \nu} \partial_{\mu} \rho \partial_{\nu} \rho-\frac{1}{2} T_{\nu} T_{\mu} G^{\mu \nu}\right) .
$$

This allows us to express $\int d^{4} x \sqrt{|G|} \mathcal{R}$ in terms of the totally antisymmetric part of the torsion. Using the on-shell relation for $T_{\mu}(3.36)$, this reduces to

$$
0=\int d^{4} x \sqrt{|G|}\left(-3 \mathcal{R}+14 \rho^{-2} G^{\mu \nu} \partial_{\mu} \rho \partial_{\nu} \rho-\frac{1}{2} \rho^{-4} G^{\mu \nu} \partial_{\mu} \tilde{\rho} \partial_{\nu} \tilde{\rho}\right) .
$$

This illustrates the fact that dilaton and axion are not independent degrees of freedom, as discussed in section 5 .

\section{E.3 Variation of the action $S_{T}$}

Consider the action term for the totally antisymmetric torsion

$$
S_{T}=\int d^{4} x \sqrt{|G|} G^{\mu \nu} T_{\mu} T_{\nu}
$$

Here $T_{\mu}$ is defined in (B.15), or explicitly

$$
T_{\rho}=\frac{1}{2} \sqrt{|G|}^{-1} \rho^{2} G_{\rho \kappa} \varepsilon^{\nu \sigma \mu \kappa} T_{\nu \sigma \mu} .
$$

Its variation can be written as

$$
2 \delta T_{\rho}=\sqrt{|G|}^{-1} \rho^{2}\left(G_{\rho \kappa} \varepsilon^{\nu \sigma \mu \kappa} \delta T_{\nu \sigma \mu}+\delta G_{\rho \kappa} \varepsilon^{\nu \sigma \mu \kappa} T_{\nu \sigma \mu}\right)+\left(\rho^{-2} \delta \rho^{2}+\frac{1}{2} \delta G^{\alpha \beta} G_{\alpha \beta}\right) 2 T_{\rho} .
$$

Using

$$
\begin{aligned}
\varepsilon^{\nu \sigma \mu \kappa} \delta T_{\nu \sigma \mu} & =\varepsilon^{\nu \sigma \mu \kappa}\left(\left(\partial_{\nu} E^{\dot{\alpha}}{ }_{\sigma}-\partial_{\sigma} E^{\dot{\alpha}}{ }_{\nu}\right) \delta E_{\dot{\alpha} \mu}+\left(\partial_{\nu} \delta E^{\dot{\alpha}}{ }_{\sigma}-\partial_{\sigma} \delta E^{\dot{\alpha}}{ }_{\nu}\right) E_{\dot{\alpha} \mu}\right) \\
& =\varepsilon^{\nu \sigma \mu \kappa}\left(T_{\nu \sigma}{ }^{\dot{\alpha}} \delta E_{\dot{\alpha} \mu}+2 \partial_{\nu} \delta E^{\dot{\alpha}}{ }_{\sigma} E_{\dot{\alpha} \mu}\right)
\end{aligned}
$$

and partial integration we obtain

$$
\begin{aligned}
\int d^{4} x & \rho^{2} T_{\kappa} \varepsilon^{\nu \mu \sigma \kappa} \delta T_{\nu \sigma \mu} \\
& =\int d^{4} x\left(\rho^{2} T_{\kappa} \varepsilon^{\nu \mu \sigma \kappa} T_{\nu \sigma}{ }^{\dot{\alpha}} \delta E_{\dot{\alpha} \mu}-2 \varepsilon^{\nu \mu \sigma \kappa} \partial_{\nu}\left(\rho^{2} T_{\kappa}\right) E_{\dot{\alpha} \mu} \delta E_{\sigma}^{\dot{\alpha}}-\rho^{2} T_{\kappa} \varepsilon^{\nu \mu \sigma \kappa} T_{\nu \mu}{ }^{\dot{\alpha}} \delta E_{\dot{\alpha} \sigma}\right) \\
& =2 \int d^{4} x \rho^{2} \delta E_{\dot{\alpha} \mu}\left(T_{\kappa} \varepsilon^{\nu \mu \sigma \kappa} T_{\nu \sigma}^{\dot{\alpha}}+E_{\sigma}^{\dot{\alpha}} \varepsilon^{\nu \mu \sigma \kappa} \rho^{-2} \partial_{\nu}\left(\rho^{2} T_{\kappa}\right)\right) .
\end{aligned}
$$


Therefore the variation of $S_{T}$ can be written as

$$
\begin{aligned}
\delta S_{T}= & \int d^{4} x \sqrt{|G|} \delta G^{\mu \nu}\left(T_{\mu} T_{\nu}-\frac{1}{2} G_{\mu \nu} T \cdot T\right)+\int d^{4} x \sqrt{|G|} G^{\mu \nu} T_{\mu} 2 \delta T_{\nu} \\
= & \int d^{4} x \sqrt{|G|} \delta G^{\mu \nu}\left(T_{\mu} T_{\nu}+\frac{1}{2} G_{\mu \nu} T \cdot T\right) \\
& +\int d^{4} x\left(\rho^{2} T_{\kappa} \varepsilon^{\nu \sigma \mu \kappa} \delta T_{\nu \sigma \mu}+\rho^{2} T_{\eta} G^{\eta \rho} \delta G_{\rho \kappa} \varepsilon^{\nu \sigma \mu \kappa} T_{\nu \sigma \mu}\right)+2 \int d^{4} x \rho^{-2} \delta \rho^{2} T \cdot T \\
= & \int d^{4} x \sqrt{|G|}\left(-\delta G^{\mu \nu}\left(T_{\mu} T_{\nu}-\frac{1}{2} G_{\mu \nu} T \cdot T\right)+2 \rho^{-2} \delta \rho^{2} T \cdot T\right)+\int d^{4} x \rho^{2} T_{\kappa} \varepsilon^{\nu \sigma \mu \kappa} \delta T_{\nu \sigma \mu} \\
= & \int d^{4} x \sqrt{|G|}\left(-\delta G^{\mu \nu}\left(T_{\mu} T_{\nu}-\frac{1}{2} G_{\mu \nu} T \cdot T\right)+2 \rho^{-2} \delta \rho^{2} T \cdot T\right) \\
& -2 \int d^{4} x \rho^{2} \delta E_{\dot{\alpha} \mu}\left(T_{\kappa} \varepsilon^{\nu \mu \sigma \kappa} T_{\nu \sigma}{ }^{\dot{\alpha}}+E^{\dot{\alpha}}{ }_{\sigma} \varepsilon^{\nu \mu \sigma \kappa} \rho^{-2} \partial_{\nu}\left(\rho^{2} T_{\kappa}\right)\right)
\end{aligned}
$$

where $T \cdot T=G^{\mu \nu} T_{\mu} T_{\nu}$. This is used in (4.3).

Open Access. This article is distributed under the terms of the Creative Commons Attribution License (CC-BY 4.0), which permits any use, distribution and reproduction in any medium, provided the original author(s) and source are credited.

\section{References}

[1] H.C. Steinacker, Higher-spin gravity and torsion on quantized space-time in matrix models, JHEP 04 (2020) 111 [arXiv:2002.02742] [INSPIRE].

[2] S. Doplicher, K. Fredenhagen and J.E. Roberts, The Quantum structure of space-time at the Planck scale and quantum fields, Commun. Math. Phys. 172 (1995) 187 [hep-th/0303037] [INSPIRE].

[3] N. Ishibashi, H. Kawai, Y. Kitazawa and A. Tsuchiya, A Large $N$ reduced model as superstring, Nucl. Phys. B 498 (1997) 467 [hep-th/9612115] [INSPIRE].

[4] H. Aoki, S. Iso, H. Kawai, Y. Kitazawa and T. Tada, Space-time structures from IIB matrix model, Prog. Theor. Phys. 99 (1998) 713 [hep-th/9802085] [InSPIRE].

[5] H. Steinacker, Emergent Geometry and Gravity from Matrix Models: an Introduction, Class. Quant. Grav. 27 (2010) 133001 [arXiv:1003.4134] [INSPIRE].

[6] S.-W. Kim, J. Nishimura and A. Tsuchiya, Expanding $(3+1)$-dimensional universe from a Lorentzian matrix model for superstring theory in (9+1)-dimensions, Phys. Rev. Lett. 108 (2012) 011601 [arXiv:1108.1540] [INSPIRE].

[7] S.-W. Kim, J. Nishimura and A. Tsuchiya, Late time behaviors of the expanding universe in the IIB matrix model, JHEP 10 (2012) 147 [arXiv:1208.0711] [INSPIRE].

[8] J. Nishimura and A. Tsuchiya, Complex Langevin analysis of the space-time structure in the Lorentzian type IIB matrix model, JHEP 06 (2019) 077 [arXiv: 1904.05919] [INSPIRE].

[9] A. Chaney, L. Lu and A. Stern, Matrix Model Approach to Cosmology, Phys. Rev. D 93 (2016) 064074 [arXiv: 1511.06816] [INSPIRE].

[10] H.C. Steinacker, Cosmological space-times with resolved Big Bang in Yang-Mills matrix models, JHEP 02 (2018) 033 [arXiv:1709.10480] [inSPIRE]. 
[11] K. Hatakeyama, A. Matsumoto, J. Nishimura, A. Tsuchiya and A. Yosprakob, The emergence of expanding space-time and intersecting D-branes from classical solutions in the Lorentzian type IIB matrix model, Prog. Theor. Exp. Phys. 2020 (2020) 043B10 [arXiv: 1911.08132] [INSPIRE].

[12] D. Klammer and H. Steinacker, Cosmological solutions of emergent noncommutative gravity, Phys. Rev. Lett. 102 (2009) 221301 [arXiv:0903.0986] [inSPIRE].

[13] M. Hanada, H. Kawai and Y. Kimura, Describing curved spaces by matrices, Prog. Theor. Phys. 114 (2006) 1295 [hep-th/0508211] [INSPIRE].

[14] M. Sperling and H.C. Steinacker, Covariant cosmological quantum space-time, higher-spin and gravity in the IKKT matrix model, JHEP 07 (2019) 010 [arXiv:1901.03522] [INSPIRE].

[15] J. Heckman and H. Verlinde, Covariant non-commutative space-time, Nucl. Phys. B 894 (2015) 58 [arXiv: 1401.1810] [INSPIRE].

[16] H.C. Steinacker, Emergent gravity on covariant quantum spaces in the IKKT model, JHEP 12 (2016) 156 [arXiv: 1606.00769] [INSPIRE].

[17] K. Hasebe, Non-Compact Hopf Maps and Fuzzy Ultra-Hyperboloids, Nucl. Phys. B $86 \mathbf{6}$ (2012) 148 [arXiv:1207.1968] [INSPIRE].

[18] M. Sperling and H.C. Steinacker, The fuzzy 4-hyperboloid $H_{n}^{4}$ and higher-spin in Yang-Mills matrix models, Nucl. Phys. B 941 (2019) 680 [arXiv:1806.05907] [INSPIRE].

[19] M.A. Vasiliev, Consistent equation for interacting gauge fields of all spins in (3+1)-dimensions, Phys. Lett. B 243 (1990) 378 [InSPIRE].

[20] V.E. Didenko and E.D. Skvortsov, Elements of Vasiliev theory, arXiv:1401.2975 [INSPIRE].

[21] H.C. Steinacker, Higher-spin kinematics $\& 3$ no ghosts on quantum space-time in Yang-Mills matrix models, arXiv:1910.00839 [INSPIRE].

[22] H.C. Steinacker, Scalar modes and the linearized Schwarzschild solution on a quantized FLRW space-time in Yang-Mills matrix models, Class. Quant. Grav. 36 (2019) 205005 [arXiv: 1905. 07255] [INSPIRE].

[23] H.C. Steinacker, On the quantum structure of space-time, gravity, and higher spin in matrix models, Class. Quant. Grav. 37 (2020) 113001 [arXiv:1911.03162] [INSPIRE].

[24] N. Seiberg and E. Witten, String theory and noncommutative geometry, JHEP 09 (1999) 032 [hep-th/9908142] [INSPIRE].

[25] G. Ishiki, Matrix Geometry and Coherent States, Phys. Rev. D 92 (2015) 046009 [arXiv: 1503.01230] [INSPIRE].

[26] H.C. Steinacker, Quantum (Matrix) Geometry and Quasi-Coherent States, J. Phys. A $\mathbf{5 4}$ (2021) 055401 [arXiv:2009.03400] [INSPIRE].

[27] H. Aoki, S. Iso, H. Kawai, Y. Kitazawa, A. Tsuchiya and T. Tada, IIB matrix model, Prog. Theor. Phys. Suppl. 134 (1999) 47 [hep-th/9908038] [InSPIRE].

[28] R. Aldrovandi and J.G. Pereira, Teleparallel gravity: an introduction, in Fundamental Theories of Physics 173, Springer, Dordrecht The Netherlands (2012) [INSPIRE].

[29] R. Penrose and W. Rindler, Spinors and space-time. Volume 1. Two-spinor calculus and relativistic fields, in Cambridge Monographs on Mathematical Physics, Cambridge University Press, Cambridge U.K. (1984) [INSPIRE]. 
[30] D.V. Galtsov, A.A. Garcia and O.V. Kechkin, Symmetries of the stationary Einstein-Maxwell dilaton - axion theory, J. Math. Phys. 36 (1995) 5023 [INSPIRE].

[31] I. Bakas, Solitons of axion - dilaton gravity, Phys. Rev. D 54 (1996) 6424 [hep-th/9605043] [INSPIRE].

[32] G. Clement and D.V. Galtsov, Stationary BPS solutions to dilaton-axion gravity, Phys. Rev. $D 54$ (1996) 6136 [hep-th/9607043] [INSPIRE].

[33] V.E. Didenko and M.A. Vasiliev, Static BPS black hole in $4 d$ higher-spin gauge theory, Phys. Lett. B 682 (2009) 305 [Erratum ibid. 722 (2013) 389] [arXiv:0906. 3898] [INSPIRE].

[34] C. Iazeolla and P. Sundell, Families of exact solutions to Vasiliev's $4 D$ equations with spherical, cylindrical and biaxial symmetry, JHEP 12 (2011) 084 [arXiv:1107.1217] [INSPIRE].

[35] A.D. Sakharov, Vacuum quantum fluctuations in curved space and the theory of gravitation, Sov. Phys. Usp. 34 (1991) 394 [Dokl. Akad. Nauk Ser. Fiz. 177 (1967) 70] [Gen. Rel. Grav. 32 (2000) 365] [Usp. Fiz. Nauk 161 (1991) 64] [INSPIRE]. 